NISTIR 7731

\title{
Bicubic B-Spline Surface Approximation of Invariant Tori
}

S. Ramamurti

David Gilsinn 
NISTIR 7731

\title{
Bicubic B-Spline Surface Approximation of Invariant Tori
}

\author{
S. Ramamurti \\ D. Gilsinn \\ Information Technology Labortory \\ Applied and Computational Mathematics Division
}

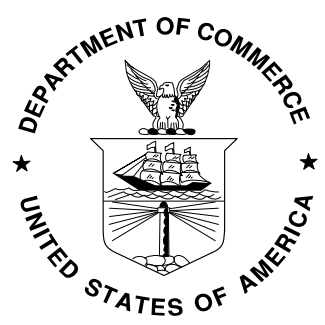

U.S. Department of Commerce Gary Locke, Secretary 


\title{
Bicubic B-Spline Surface Approximation of Invariant Tori
}

\author{
Sita Ramamurti* \\ David E. Gilsinn ${ }^{\dagger}$
}

October 14, 2010

\begin{abstract}
The invariant torus of a coupled system of Van der Pol oscillators is approximated using bicubic B-splines. The paper considers the case of strong nonlinear coupling. In particular, the shapes of invariant torii for the Van der Pol coupling parameter $\lambda$ are computed in the range $[0.1$, 2.0]. Comparisons are given with results obtained by the MATLAB differential equation solver ode45. Very good normed residual errors of the determining equations for the approximate tori for the cases $\lambda=0.1,0.6$ are shown. At the upper limit of $\lambda=2.0$ memory errors occured during the optimization phase for solving the determining equations so that full optimization for some knot sets was not achieved, but a visual comparison of the resulting invariant torus figure showed a close similarity to the solution using ode45.
\end{abstract}

Keywords: bicubic B-splines; determining equations; invariant torus; large parameter case; optimization; Van der Pol oscillators

\section{Introduction}

Physical problems involving coupled vibrating systems often lead to ordinary differential equations of the form

$$
\ddot{\mathbf{x}}+\mathbf{A x}=\lambda \mathbf{N}(\mathbf{x})
$$

where $\mathbf{x} \in \mathbf{R}^{n}$, $\mathbf{A}$ is diagonal, and $\mathbf{N}$ is a nonlinear function of $\mathbf{x} . \quad \lambda$ is a parameter that scales the nonlinearity. There are many forms of solutions to systems like (1), but we are interested in approximating a particular class of solutions. In particular, we are interested in systems that exhibit an invariant surface of solutions. That is a surface on which, if a solution passes through a point on the surface, then the entire solution remains on the surface. In the case of periodic solutions the surface is sometimes referred to as an invariant torus. In the case of small parameters there is a large literature on the existence and approximation of invariant tori (see e.g. Bogoliubov and Mitropolsky [1], Hale [9, 10], and Gilsinn [5]). For the case of a large parameter there is not much in the way of literature. The work of Ge and Leung [4] showed that the Fast Fourier Transform could be used as a tool to approximate invariant tori in the large nonlinearity case. In this study we introduce a bicubic B-spline computational algorithm that, at least for a certain class of nonlinear systems, computes invariant tori to a high degree of accuracy for a larger range of parameters. In

\footnotetext{
*Department of Mathematics, Trinity Washington University, Washington D. C. 20017, ramamurtis@trinitydc.edu

${ }^{\dagger}$ Applied and Computational Mathematics Division, Information Technology Laboratory, National Institute of Standards and Technology, Gaithersburg MD 20899-8910, david.gilsinn@nist.gov.
} 
particular, following the work of Gilsinn [6,7], we shall consider the system of coupled Van der Pol oscillators

$$
\begin{aligned}
& \ddot{x_{1}}+\mu_{1}^{2} x_{1}=\lambda\left(1-x_{1}^{2}-\alpha_{1} x_{2}^{2}\right) \dot{x_{1}}, \\
& \ddot{x_{2}}+\mu_{2}^{2} x_{2}=\lambda\left(1-\alpha_{2} x_{1}^{2}-x_{2}^{2}\right) \dot{x_{2}},
\end{aligned}
$$

where $\mu_{1} / \mu_{2}$ are irrational multiples.

Under the change of coordinates $x_{1}=r_{1}^{1 / 2} \sin \left(\mu_{1} \omega_{1}\right), \dot{x_{1}}=\mu_{1} r_{1}{ }^{1 / 2} \cos \left(\mu_{1} \omega_{1}\right), x_{2}=r_{2}^{1 / 2} \sin \left(\mu_{2} \omega_{2}\right)$, and $\dot{x_{2}}=\mu_{2} r_{2}{ }^{1 / 2} \cos \left(\mu_{2} \omega_{2}\right)$ this system becomes

$$
\begin{aligned}
\dot{\omega_{1}=} & 1+\lambda\left(-\frac{1}{\mu_{1}}\right)\left(\sin \left(\mu_{1} \omega_{1}\right) \cos \left(\mu_{1} \omega_{1}\right)-r_{1} \sin ^{3}\left(\mu_{1} \omega_{1}\right) \cos \left(\mu_{1} \omega_{1}\right)\right. \\
& \left.-\alpha_{1} r_{2} \sin \left(\mu_{1} \omega_{1}\right) \cos \left(\mu_{1} \omega_{1}\right) \sin ^{2}\left(\mu_{2} \omega_{2}\right)\right), \\
\dot{\omega_{2}=} & 1+\lambda\left(-\frac{1}{\mu_{2}}\right)\left(\sin \left(\mu_{2} \omega_{2}\right) \cos \left(\mu_{2} \omega_{2}\right)\right. \\
& \left.-\alpha_{2} r_{1} \sin ^{2}\left(\mu_{1} \omega_{1}\right) \sin \left(\mu_{2} \omega_{2}\right) \cos \left(\mu_{2} \omega_{2}\right)-r_{2} \sin ^{3}\left(\mu_{2} \omega_{2}\right)\right) \cos \left(\mu_{2} \omega_{2}\right), \\
\dot{r_{1}}= & 2 \lambda r_{1}\left(\cos ^{2}\left(\mu_{1} \omega_{1}\right)-r_{1} \sin ^{2}\left(\mu_{1} \omega_{1}\right) \cos ^{2}\left(\mu_{1} \omega_{1}\right)\right. \\
& \left.-\alpha_{1} r_{2} \cos ^{2}\left(\mu_{1} \omega_{1}\right) \sin ^{2}\left(\mu_{2} \omega_{2}\right)\right), \\
\dot{r_{2}=} & 2 \lambda r_{2}\left(\cos ^{2}\left(\mu_{2} \omega_{2}\right)-\alpha_{2} r_{1} \sin ^{2}\left(\mu_{1} \omega_{1}\right) \cos ^{2}\left(\mu_{2} \omega_{2}\right)\right. \\
& \left.-r_{2} \sin ^{2}\left(\mu_{2} \omega_{2}\right) \cos ^{2}\left(\mu_{2} \omega_{2}\right)\right),
\end{aligned}
$$

which is of the form

$$
\begin{aligned}
\dot{\boldsymbol{\Omega}} & =\mathbf{d}+\lambda \boldsymbol{\Theta}(\boldsymbol{\Omega}, \mathbf{r}), \\
\dot{\mathbf{r}} & =\lambda \mathbf{R}(\boldsymbol{\Omega}, \mathbf{r}),
\end{aligned}
$$

with $\boldsymbol{\Omega}=\left(\begin{array}{c}\omega_{1} \\ \omega_{2}\end{array}\right), \mathbf{r}=\left(\begin{array}{c}r_{1} \\ r_{2}\end{array}\right), \mathbf{d}=\left(\begin{array}{c}1 \\ 1\end{array}\right)$,

$$
\begin{gathered}
\boldsymbol{\Theta}(\boldsymbol{\Omega}, \mathbf{r})=\left(\begin{array}{l}
\Theta_{1}(\boldsymbol{\Omega}, \mathbf{r}) \\
\Theta_{2}(\boldsymbol{\Omega}, \mathbf{r})
\end{array}\right)=\left(\begin{array}{l}
\left(-\frac{1}{\mu_{1}}\right)\left(\sin \left(\mu_{1} \omega_{1}\right) \cos \left(\mu_{1} \omega_{1}\right)\right. \\
-r_{1} \sin ^{3}\left(\mu_{1} \omega_{1}\right) \cos \left(\mu_{1} \omega_{1}\right) \\
\left.-\alpha_{1} r_{2} \sin \left(\mu_{1} \omega_{1}\right) \cos \left(\mu_{1} \omega_{1}\right) \sin ^{2}\left(\mu_{2} \omega_{2}\right)\right) \\
\left(-\frac{1}{\mu_{2}}\right)\left(\sin \left(\mu_{2} \omega_{2}\right) \cos \left(\mu_{2} \omega_{2}\right)\right. \\
-\alpha_{2} r_{1} \sin ^{2}\left(\mu_{1} \omega_{1}\right) \sin \left(\mu_{2} \omega_{2}\right) \cos \left(\mu_{2} \omega_{2}\right) \\
-r_{2} \sin ^{3}\left(\mu_{2} \omega_{2}\right) \cos \left(\mu_{2} \omega_{2}\right)
\end{array}\right), \\
\mathbf{R}(\boldsymbol{\Omega}, \mathbf{r})=\left(\begin{array}{l}
R_{1}(\boldsymbol{\Omega}, \mathbf{r}) \\
R_{2}(\boldsymbol{\Omega}, \mathbf{r})
\end{array}\right)=\left(\begin{array}{l}
2 r_{1}\left(\cos ^{2}\left(\mu_{1} \omega_{1}\right)\right. \\
-r_{1} \sin ^{2}\left(\mu_{1} \omega_{1}\right) \cos ^{2}\left(\mu_{1} \omega_{1}\right) \\
\left.-\alpha_{1} r_{2} \cos ^{2}\left(\mu_{1} \omega_{1}\right) \sin ^{2}\left(\mu_{2} \omega_{2}\right)\right) \\
2 r_{2}\left(\cos ^{2}\left(\mu_{2} \omega_{2}\right)\right. \\
-\alpha_{2} r_{1} \sin ^{2}\left(\mu_{1} \omega_{1}\right) \cos ^{2}\left(\mu_{2} \omega_{2}\right) \\
\left.-r_{2} \sin ^{2}\left(\mu_{2} \omega_{2}\right) \cos ^{2}\left(\mu_{2} \omega_{2}\right)\right)
\end{array}\right) .
\end{gathered}
$$


For the current study we will assume that an invariant torus exists for (3) in the form

$$
\begin{gathered}
S=\left\{\left(\mu_{1} \omega_{1}, \mu_{2} \omega_{2}, r_{1}, r_{2}\right): r_{1}=f\left(\mu_{1} \omega_{1}, \mu_{2} \omega_{2}\right), r_{2}=g\left(\mu_{1} \omega_{1}, \mu_{2} \omega_{2}\right),\right. \\
\left.\left(\mu_{1} \omega_{1}, \mu_{2} \omega_{2}\right) \in T^{2}\right\} .
\end{gathered}
$$

Using the invariance of the graphs of $f\left(\mu_{1} \omega_{1}, \mu_{2} \omega_{2}\right)$ and $g\left(\mu_{1} \omega_{1}, \mu_{2} \omega_{2}\right)$ under the dynamics generated by (3) we have

$$
\begin{aligned}
\dot{\mathbf{r}} & =D_{\omega_{1}} \mathbf{F}\left(\mu_{1} \omega_{1}, \mu_{2} \omega_{2}\right) \dot{\omega}_{1}+D_{\omega_{2}} \mathbf{F}\left(\mu_{1} \omega_{1}, \mu_{2} \omega_{2}\right) \dot{\omega}_{2} \\
& =\lambda \mathbf{R}\left(\boldsymbol{\Omega}, \mathbf{F}\left(\mu_{1} \omega_{1}, \mu_{2} \omega_{2}\right)\right),
\end{aligned}
$$

where $\mathbf{F}=\left(\begin{array}{c}f \\ g\end{array}\right)$. Now, from (4),

$$
\begin{aligned}
& \dot{\omega}_{1}=1+\lambda \Theta_{1}\left(\boldsymbol{\Omega}, \mathbf{F}\left(\mu_{1} \omega_{1}, \mu_{2} \omega_{2}\right)\right), \\
& \dot{\omega_{2}}=1+\lambda \Theta_{2}\left(\boldsymbol{\Omega}, \mathbf{F}\left(\mu_{1} \omega_{1}, \mu_{2} \omega_{2}\right)\right) .
\end{aligned}
$$

We therefore substitute (7) into (6) to get the following quasilinear partial differential equation that $\mathbf{F}\left(\mu_{1} \omega_{1}, \mu_{2} \omega_{2}\right)$ must satisfy in order for its graph to be an invariant torus (See [8], [13]).

$$
\begin{aligned}
N\left(\mathbf{F}\left(\mu_{1} \omega_{1}, \mu_{2} \omega_{2}\right)\right) & =D_{\omega_{1}} \mathbf{F}\left(\mu_{1} \omega_{1}, \mu_{2} \omega_{2}\right)\left(1+\lambda \Theta_{1}\left(\boldsymbol{\Omega}, \mathbf{F}\left(\mu_{1} \omega_{1}, \mu_{2} \omega_{2}\right)\right)\right) \\
& +D_{\omega_{2}} \mathbf{F}\left(\mu_{1} \omega_{1}, \mu_{2} \omega_{2}\right)\left(1+\lambda \Theta_{2}\left(\boldsymbol{\Omega}, \mathbf{F}\left(\mu_{1} \omega_{1}, \mu_{2} \omega_{2}\right)\right)\right) \\
& -\lambda \mathbf{R}\left(\boldsymbol{\Omega}, \mathbf{F}\left(\mu_{1} \omega_{1}, \mu_{2} \omega_{2}\right)\right)=0 .
\end{aligned}
$$

For the remainder of the discussion, we make the following substitutions in order to simplify the notation in the equations.

$$
\begin{aligned}
\theta & =\mu_{1} \omega_{1}, \\
\phi & =\mu_{2} \omega_{2} .
\end{aligned}
$$

\section{Bicubic Spline Collocation Method}

We now use the method of collocation with B-splines to approximate the functions $r_{1}=f(\theta, \phi)$ and $r_{2}=g(\theta, \phi)$. We let

$$
\begin{aligned}
& 0=\theta_{0}<\theta_{1}<\ldots<\theta_{n}=2 \pi, \\
& 0=\phi_{0}<\phi_{1}<\ldots<\phi_{m}=2 \pi,
\end{aligned}
$$

be a uniform partition of the rectangle ranging over $[0,2 \pi]$ in the $\theta$ direction and $[0,2 \pi]$ in the $\phi$ direction where $n$ and $m$ are the number of knots in the $\theta$ and $\phi$ directions, respectively and $\Delta h=\theta_{i+1}-\theta_{i}=2 \pi / n, i=0, \ldots, n-1$ and $\Delta k=\phi_{j+1}-\phi_{j}=2 \pi / m, j=0, \ldots, m-1$. We seek bicubic spline surfaces of the form

$$
\begin{aligned}
r_{1, n m}(\theta, \phi) & =\sum_{i=-1}^{n+1} \sum_{j=-1}^{m+1} c_{i j} B_{i}(\theta) B_{j}(\phi), \\
r_{2, n m}(\theta, \phi) & =\sum_{i=-1}^{n+1} \sum_{j=-1}^{m+1} d_{i j} B_{i}(\theta) B_{j}(\phi),
\end{aligned}
$$


where $\left\{B_{-1}(\theta), B_{0}(\theta), \ldots, B_{n+1}(\theta)\right\}$ are the $\mathrm{B}$ spline basis functions in the $\theta$ direction with knots $\theta_{h}, h=0, \ldots, n$ and $\left\{B_{-1}(\phi), B_{0}(\phi), \ldots, B_{m+1}(\phi)\right\}$ are the $\mathrm{B}$ spline basis functions in the $\phi$ direction with knots $\phi_{k}, k=0, \ldots, m$. The functions $B_{i}(\theta)$ are defined in Prenter[12] as follows by introducing four additional knots in each direction satisfying

$$
\begin{aligned}
& \theta_{-2}<\theta_{-1}<\theta_{0}=0 \text { and } \theta_{n+2}>\theta_{n+1}>\theta_{n}=2 \pi \text {, } \\
& \phi_{-2}<\phi_{-1}<\phi_{0}=0 \text { and } \phi_{n+2}>\phi_{n+1}>\phi_{n}=2 \pi \text {, } \\
& B_{i}(\theta)=\frac{1}{h^{3}}\left\{\begin{array}{lr}
\left(\theta-\theta_{i-2}\right)^{3} & \text { if } \theta \in\left[\theta_{i-2}, \theta_{i-1}\right] \\
h^{3}+3 h^{2}\left(\theta-\theta_{i-1}\right)+3 h\left(\theta-\theta_{i-1}\right)^{2}-3\left(\theta-\theta_{i-1}\right)^{3} & \text { if } \theta \in\left[\theta_{i-1}, \theta_{i}\right] \\
h^{3}+3 h^{2}\left(\theta_{i+1}-\theta\right)+3 h\left(\theta_{i+1}-\theta\right)^{2}-3\left(\theta_{i+1}-\theta\right)^{3} \\
0 & \text { if } \theta \in\left[\theta_{i}, \theta_{i+1}\right] \\
0 & \text { otherwise. }
\end{array}\right.
\end{aligned}
$$

The function $B_{j}(\phi)$ is defined similarly. These functions are twice continuously differentiable on the real line. We can therefore define $B_{i}^{\prime}(\theta)$ and $B_{i}^{\prime \prime}(\theta)$ using the equations for $B_{i}(\theta)$. To compute the approximations $r_{1, n m}$ and $r_{2, n m}$ in (9) at the knots, we note that by substituting the knots $\theta_{h}$ and $\phi_{k}$ into the definitions of $B, B^{\prime}, B^{\prime \prime}$,

$$
B_{i}\left(\theta_{h}\right) B_{j}\left(\phi_{k}\right)= \begin{cases}16 \quad \text { if } h=i, k=j \\ 4 \quad \text { if } h=i-1, k=j \quad \text { or } \quad h=i, k=j-1 \\ \quad \text { or } h=i, k=j+1 \quad \text { or } \quad h=i+1, k=j, \\ 1 \quad \text { if } h=i-1, k=j-1 \\ \text { or } h=i-1, k=j+1 \\ \text { or } h=i+1, k=j-1 \\ \text { or } h=i+1, k=j+1,\end{cases}
$$

and $B_{i}\left(\theta_{h}\right) B_{j}\left(\phi_{k}\right) \equiv 0 \quad$ for $\quad h \geq i+2, k \geq j+2 \quad$ and $\quad h \leq i-2, k \leq j-2$.

The partial derivatives of the interpolants $r_{1, n m}(\theta, \phi), r_{2, n m}(\theta, \phi)$ at the knots can be easily found using the first derivatives and the products $B_{i}^{\prime}\left(\theta_{h}\right) B_{j}\left(\phi_{k}\right)$ and $B_{i}\left(\theta_{h}\right) B_{j}^{\prime}\left(\phi_{k}\right)$ of the spline functions in the tables below. 


\begin{tabular}{cccc}
\hline & $\left(\theta_{i-1}, \phi_{j-1}\right)$ & $\left(\theta_{i-1}, \phi_{j}\right)$ & $\left(\theta_{i-1}, \phi_{j+1}\right)$ \\
\hline$B_{i}^{\prime}(\theta) B_{j}(\phi)$ & $\frac{3}{\Delta h}$ & $\frac{12}{\Delta h}$ & $\frac{3}{\Delta h}$ \\
$B_{i}(\theta) B_{j}^{\prime}(\phi)$ & $\frac{3}{\Delta k}$ & 0 & $-\frac{3}{\Delta k}$ \\
\hline & $\left(\theta_{i}, \phi_{j-1}\right)$ & $\left(\theta_{i}, \phi_{j}\right)$ & $\left(\theta_{i}, \phi_{j+1}\right)$ \\
\hline$B_{i}^{\prime}(\theta) B_{j}(\phi)$ & 0 & 0 & 0 \\
$B_{i}(\theta) B_{j}^{\prime}(\phi)$ & $\frac{12}{\Delta k}$ & 0 & $-\frac{12}{\Delta k}$ \\
\hline & & & \\
\hline & & & \\
\hline$B_{i}^{\prime}(\theta) B_{j}(\phi)$ & $-\frac{3}{\Delta h}$ & $-\frac{12}{\Delta h}$ & $-\frac{3}{\Delta h}$ \\
$B_{i}(\theta) B_{j}^{\prime}(\phi)$ & $\frac{3}{\Delta k}$ & 0 & $-\frac{3}{\Delta k}$ \\
\hline
\end{tabular}

Table 1: Partial derivatives of the interpolants $r_{1, n m}(\theta, \phi), r_{2, n m}(\theta, \phi)$ at knots

Using the above information, the values of the approximations $r_{1, n m}$ and $r_{2, n m}$ at the knots can be expressed in terms of the parameters $c_{i j}$ and $d_{i j}$ as

$$
\begin{aligned}
r_{1, n m}\left(\theta_{h}, \phi_{k}\right)= & 16 c_{h, k}+4\left(c_{h-1, k}+c_{h, k-1}+c_{h, k+1}+c_{h+1, k}\right) \\
& +c_{h-1, k-1}+c_{h-1, k+1}+c_{h+1, k-1}+c_{h+1, k+1}, \\
r_{2, n m}\left(\theta_{h}, \phi_{k}\right)= & 16 d_{h, k}+4\left(d_{h-1, k}+d_{h, k-1}+d_{h, k+1}+d_{h+1, k}\right) \\
& +d_{h-1, k-1}+d_{h-1, k+1}+d_{h+1, k-1}+d_{h+1, k+1}
\end{aligned}
$$

A similar computation shows that the derivatives $\frac{\partial r_{1, n m}}{\partial \theta}\left(\theta_{h}, \phi_{k}\right), \frac{\partial r_{1, n m}}{\partial \phi}\left(\theta_{h}, \phi_{k}\right), \frac{\partial r_{2, n m}}{\partial \theta}\left(\theta_{h}, \phi_{k}\right)$ and $\frac{\partial r_{2, n m}}{\partial \phi}\left(\theta_{h}, \phi_{k}\right)$ at the knots have the following representation in terms of the parameters.

$$
\begin{aligned}
\frac{\partial r_{1, n m}}{\partial \theta}\left(\theta_{h}, \phi_{k}\right)= & \frac{3}{\Delta h}\left(c_{h+1, k+1}+4 c_{h+1, k}+c_{h+1, k-1}\right. \\
& \left.-c_{h-1, k+1}-4 c_{h-1, k}-c_{h-1, k-1}\right), \\
\frac{\partial r_{1, n m}}{\partial \phi}\left(\theta_{h}, \phi_{k}\right)= & \frac{3}{\Delta k}\left(c_{h+1, k+1}-c_{h+1, k-1}+4 c_{h, k+1}\right. \\
& \left.-4 c_{h, k-1}+c_{h-1, k+1}-c_{h-1, k-1}\right),
\end{aligned}
$$




$$
\begin{aligned}
\frac{\partial r_{2, n m}}{\partial \theta}\left(\theta_{h}, \phi_{k}\right)= & \frac{3}{\Delta h}\left(d_{h+1, k+1}+4 d_{h+1, k}+d_{h+1, k-1}\right. \\
& \left.-d_{h-1, k+1}-4 d_{h-1, k}-d_{h-1, k-1}\right) \\
\frac{\partial r_{2, n m}}{\partial \phi}\left(\theta_{h}, \phi_{k}\right)= & \frac{3}{\Delta k}\left(d_{h+1, k+1}-d_{h+1, k-1}+4 d_{h, k+1}\right. \\
& \left.-4 d_{h, k-1}+d_{h-1, k+1}-d_{h-1, k-1}\right) .
\end{aligned}
$$

To apply the collocation method, we choose the collocation points to coincide with the knots and then substitute the nodal values $r_{1, n m}\left(\theta_{h}, \phi_{k}\right), r_{2, n m}\left(\theta_{h}, \phi_{k}\right)$ and their derivatives obtained in equations (13) and (14) into the partial differential equation (8). This results in the following system of $(n+1) \times(m+1)$ coupled nonlinear equations in $(n+3) \times(m+3)$ parameters $c_{i j}$ and $d_{i j}$ :

$$
\begin{array}{r}
{\left[\frac { 3 } { \Delta h } \left(c_{h+1, k+1}+4 c_{h+1, k}+c_{h+1, k-1}-c_{h-1, k+1}\right.\right.} \\
\left.\left.-4 c_{h-1, k}-c_{h-1, k-1}\right)\right]\left(\left(1+\lambda \Theta_{1}\left(\boldsymbol{\Omega}, r_{1, n m}\left(\theta_{h}, \phi_{k}\right)\right)\right)\right) \\
+\left[\frac { 3 } { \Delta k } \left(c_{h+1, k+1}-c_{h+1, k-1}+4 c_{h, k+1}-4 c_{h, k-1}\right.\right. \\
\left.\left.+c_{h-1, k+1}-c_{h-1, k-1}\right)\right]\left(1+\lambda \Theta_{2}\left(\boldsymbol{\Omega}, r_{1, n m}\left(\theta_{h}, \phi_{k}\right)\right)\right) \\
-\lambda \mathbf{R}\left(\boldsymbol{\Omega}, r_{1, n m}\left(\theta_{h}, \phi_{k}\right)\right)=0,
\end{array}
$$

for $h=0, \ldots, n$ and $k=0, \ldots, m$.

\section{Periodicity Conditions}

The surface we are approximating is a closed one generated by two periodic closed curves. The spline model is therefore chosen so that it is closed in both the $\theta$ and $\phi$ directions. This is accomplished by repeating both the knots and the control vertices as follows.

$$
\begin{array}{ll}
\left(\theta_{n}, \phi_{k}\right)=\left(\theta_{0}, \phi_{k}\right), & 0 \leq k \leq m, \quad\left(\theta_{h}, \phi_{m}\right)=\left(\theta_{h}, \phi_{0}\right), \quad 0 \leq h \leq n \\
c_{-1, j}=c_{n-1, j} & -1 \leq j \leq m+1 \\
c_{n+1, j}=c_{1, j}, & -1 \leq i \leq n+1 \\
c_{i,-1}=c_{i, m-1} & 0 \leq k \leq m \\
c_{i, m+1}=c_{i, 1}, & 0 \leq h \leq n \\
c_{n, k}=c_{0, k}, &
\end{array}
$$

Application of the above periodic boundary conditions reduces equation (13) to a $\mathrm{nm} \times \mathrm{nm}$ block- 
pentadiagonal matrix equation of the form

$$
\left[\begin{array}{c}
r_{1, n m}(0,0) \\
r_{1, n m}(0,1) \\
r_{1, n m}(0,2) \\
\vdots \\
r_{1, n m}((n-1),(m-2)) \\
r_{1, n m}((n-1),(m-1))
\end{array}\right]=\left[\begin{array}{cccccc}
D & B & 0 & \ldots & 0 & B \\
B & D & B & 0 & \ldots & 0 \\
0 & B & D & B & \ldots & 0 \\
\vdots & \vdots & \vdots & \vdots & \vdots & \vdots \\
0 & \ldots & 0 & B & D & B \\
B & 0 & \ldots & 0 & B & D
\end{array}\right]\left[\begin{array}{c}
c_{0,0} \\
c_{0,1} \\
c_{0,2} \\
\vdots \\
c_{(n-1),(m-2)} \\
c_{(n-1),(m-1)}
\end{array}\right]
$$

where $\mathrm{D}$ and $\mathrm{B}$ are $m \times m$ pentadiagonal matrices with entries

$$
D=\left[\begin{array}{cccccc}
16 & 4 & 0 & \ldots & 0 & 4 \\
4 & 16 & 4 & 0 & \ldots & 0 \\
0 & 4 & 16 & 4 & \ldots & 0 \\
\vdots & \vdots & \vdots & \vdots & \vdots & \vdots \\
0 & \ldots & 0 & 4 & 16 & 4 \\
4 & 0 & \ldots & 0 & 4 & 16
\end{array}\right]
$$

and

$$
B=\left[\begin{array}{cccccc}
4 & 1 & 0 & \ldots & 0 & 1 \\
1 & 4 & 1 & 0 & \ldots & 0 \\
0 & 1 & 4 & 1 & \ldots & 0 \\
\vdots & \vdots & \vdots & \vdots & \vdots & \vdots \\
0 & \ldots & 0 & 1 & 4 & 1 \\
1 & 0 & \ldots & 0 & 1 & 4
\end{array}\right]
$$

with a similar matrix equation obtained for the approximation $r_{2, n m}$ by replacing the control points $c_{i, j}$ with $d_{i, j}$.

Similarly the system of partial derivatives of $r_{1, n m}$ in (14) reduce, after applying the periodic boundary conditions, to a block diagonal form

$$
\left[\begin{array}{c}
\frac{\partial r_{1, n m}}{\partial \theta}(0,0) \\
\frac{\partial r_{1, n m}}{\partial \theta}(0,1) \\
\frac{\partial r_{1, n m}}{\partial \theta}(0,2) \\
\vdots \\
\frac{\partial r_{1, n m}}{\partial \theta}((n-1),(m-2)) \\
\frac{\partial r_{1, n m}}{\partial \theta}((n-1),(m-1))
\end{array}\right]=\left[\begin{array}{cccccc}
0 & B & 0 & \ldots & 0 & -B \\
-B & 0 & B & 0 & \cdots & 0 \\
0 & -B & 0 & B & \cdots & 0 \\
\vdots & \vdots & \vdots & \vdots & \vdots & \vdots \\
0 & \cdots & 0 & -B & 0 & B \\
B & 0 & \ldots & 0 & -B & 0
\end{array}\right]\left[\begin{array}{c}
c_{0,0} \\
c_{0,1} \\
c_{0,2} \\
\vdots \\
c_{(n-1),(m-2)} \\
c_{(n-1),(m-1)}
\end{array}\right],
$$

where $\mathrm{B}$ is a $m \times m$ diagonal matrix with entries

$$
B=\frac{3}{\Delta h}\left[\begin{array}{cccccc}
4 & 1 & 0 & \ldots & 0 & 1 \\
1 & 4 & 1 & 0 & \ldots & 0 \\
0 & 1 & 4 & 1 & \ldots & 0 \\
\vdots & \vdots & \vdots & \vdots & \vdots & \vdots \\
0 & \ldots & 0 & 1 & 4 & 1 \\
1 & 0 & \ldots & 0 & 1 & 4
\end{array}\right]
$$


and

$$
\left[\begin{array}{c}
\frac{\partial r_{1, n m}}{\partial \phi}(0,0) \\
\frac{\partial r_{1, n m}}{\partial \phi}(0,1) \\
\frac{\partial r_{1, n m}}{\partial \phi}(0,2) \\
\vdots \\
\frac{\partial r_{1, n m} \partial}{\partial \phi}((n-1),(m-2)) \\
\frac{\partial r_{1, n m}}{\partial \phi}((n-1),(m-1))
\end{array}\right]=\left[\begin{array}{cccccc}
D & B & 0 & \ldots & 0 & B \\
B & D & B & 0 & \ldots & 0 \\
0 & B & D & B & \ldots & 0 \\
\vdots & \vdots & \vdots & \vdots & \vdots & \vdots \\
0 & \ldots & 0 & B & D & B \\
B & 0 & \ldots & 0 & B & D
\end{array}\right]\left[\begin{array}{c}
c_{0,0} \\
c_{0,1} \\
c_{0,2} \\
\vdots \\
c_{(n-1),(m-2)} \\
c_{(n-1),(m-1)}
\end{array}\right],
$$

where $\mathrm{D}$ and $\mathrm{B}$ are $m \times m$ diagonal matrices with entries

$$
D=\frac{12}{\Delta k}\left[\begin{array}{cccccc}
0 & 1 & 0 & \ldots & 0 & -1 \\
-1 & 0 & 1 & 0 & \ldots & 0 \\
0 & -1 & 0 & 1 & \ldots & 0 \\
\vdots & \vdots & \vdots & \vdots & \vdots & \vdots \\
0 & \ldots & 0 & -1 & 0 & 1 \\
1 & 0 & \ldots & 0 & -1 & 0
\end{array}\right]
$$

and

$$
B=\frac{3}{\Delta k}\left[\begin{array}{cccccc}
0 & 1 & 0 & \ldots & 0 & -1 \\
-1 & 0 & 1 & 0 & \ldots & 0 \\
0 & -1 & 0 & 1 & \ldots & 0 \\
\vdots & \vdots & \vdots & \vdots & \vdots & \vdots \\
0 & \ldots & 0 & -1 & 0 & 1 \\
1 & 0 & \ldots & 0 & -1 & 0
\end{array}\right]
$$

with similar expressions obtained for the partial derivative approximations $\frac{\partial r_{2, n m}}{\partial \theta}, \frac{\partial r_{2, n m}}{\partial \phi}$ by replacing the control points $c_{i, j}$ with $d_{i, j}$.

\section{MATLAB solution}

We now describe the algorithmic details involved in computing the bicubic B-spline coefficients $c_{i j}$ and $d_{i j}$ by setting up an appropriate objective function to be mimimized using MATLAB's nonlinear minimization subroutine. Our objective function simply computes the "residual error": the difference between the derivative of the approximations $r_{1, n m}, r_{2, n m}$ and the derivatives $\dot{r_{1}}, \dot{r_{2}}$ respectively as given by the system (3) which is equivalent to the partial differential equation given by (8). Substituting equations (16),(17), and (18) into the partial differential equation system (15), we reduce it to a $2 \mathrm{~nm}$ system of coupled nonlinear equations in $2 \mathrm{~nm}$ parameters $c_{i j}$ and $d_{i j}$. The determining equations can now be written as

$$
\begin{array}{r}
R_{1, h k}(A)=\frac{\partial r_{1, n m}}{\partial \theta}(h, k)\left(\left(1+\lambda \Theta_{1}(\boldsymbol{\Omega}, \mathbf{r}(h, k))\right)\right)+\frac{\partial r_{1, n m}}{\partial \phi}(h, k)\left(1+\lambda \Theta_{2}(\boldsymbol{\Omega}, \mathbf{r}(h, k))\right) \\
-\lambda \mathbf{R}_{\mathbf{1}}(\boldsymbol{\Omega}, \mathbf{r}(h, k))=0, \\
R_{2, h k}(A)=\frac{\partial r_{2, n m}}{\partial \theta}(h, k)\left(\left(1+\lambda \Theta_{1}(\boldsymbol{\Omega}, \mathbf{r}(h, k))\right)\right)+\frac{\partial r_{2, n m}}{\partial \phi}(h, k)\left(1+\lambda \Theta_{2}(\boldsymbol{\Omega}, \mathbf{r}(h, k))\right)
\end{array}
$$




$$
-\lambda \mathbf{R}_{\mathbf{2}}(\boldsymbol{\Omega}, \mathbf{r}(h, k))=0
$$

for $h=0,1, \ldots, n, k=0,1, \ldots, m$, where $\mathbf{A}=[\mathbf{c} ; \mathbf{d}]$ with $\mathbf{c}=\left(c_{-1,-1}, c_{-1,0}, \ldots, c_{n+1, m+1}\right)^{T}$ and $\mathbf{d}=\left(d_{-1,-1}, d_{-1,0}, \ldots, d_{n+1, m+1}\right)^{T}$. Equation (19) can then be used to find an approximation for the vector $\mathbf{A}$ by a minimization process, such as

$$
\min _{c, d} \sum_{k=1}^{2 n m}\left|R_{k}(A)\right|^{2}
$$

We use MATLAB's fsolve command to solve the system of nonlinear equations for the parameters. For an initial guess, we fix the following parameters for this case study:

$$
\alpha_{1}=0.2, \alpha_{2}=0.4, \mu_{1}=1, \mu_{2}=1.414
$$

and take the first approximation to the radii as

$$
r_{1, n m}=3.53, r_{2, n m}=1.18
$$

We note that the matrix form of equation (16) is

$$
A c=r,
$$

with the matrix A being nonsingular. That is, system (16) has a unique solution and consequently, the collocation approximate (9)satisfying the periodic boundary conditions is uniquely defined. We can therefore solve (23) by substituting the initial guess for $r_{1, n m}$ and $r_{2, n m}$ as 3.53 and 1.18 respectively into the right hand side of (23)to obtain the vector $c$ of the unknown parameters $c_{i j}$ as

$$
c=A^{-1} r .
$$

A similar calculation yields the initial vector $d$ of the unknown parameters $d_{i j}$.

\section{Optimization Issues}

For the first few runs, we coded the objective function and called up the fsolve optimization routine without supplying the sparsity pattern of the Jacobian. This worked well for small values of $\lambda$ but as the strength of coupling increased, we had to increase the size of the knot domain for getting a good approximation as well as for the optimization to terminate successfully. Increasing the size of the knot domain for bigger values of $\lambda$ slowed down the optimization considerably. Computationally, the model proved expensive even for $\lambda=0.5$, which appeared to require a 40 by 40 knot domain for a reasonably good approximation.

The residual error for any point in the knot domain depends only on the nine surrounding parameters and therefore has very few non-zero partial derivatives. The Jacobian is sparse with well-structured blocks of non-zero elements. The computational cost of finite differencing, which MATLAB uses to approximate the Jacobian, can therefore be reduced significantly, if we can supply 
the structure of the Jacobian to MATLAB. This is done by setting JacobPattern to 'on' in options. This was our first improvement to the code. With this enhancement the results were encouraging for $\lambda$ between 0.1 and 1.0: The number of function evaluations per iteration dropped significantly (for example, for $\lambda=0.5$ with a 40 by 40 knot domain, the number of evaluations dropped from 3202 to just 32 per iteration!), and the time required for the optimization to successfully terminate reduced considerably. The following table summarizes these results. Unfortunately, supplying the

\begin{tabular}{|c|c|c|}
\hline \multicolumn{3}{|c|}{$\lambda=0.5, \quad$ knots $=40$} \\
\hline Implementation & Normalized Residual Error & Time for Optimization \\
\hline Jacobian Structure Unspecified & $2.26395 \mathrm{e}-007$ & $4.0188 \mathrm{e}+003$ secs \\
\hline Jacobian Structure Specified & $2.33638 \mathrm{e}-007$ & 41.3750 secs \\
\hline
\end{tabular}

Jacobian Sparsity Pattern appears to be insufficient for $\lambda$ bigger than or equal to 1.0, which require bigger knot domains for achieving the required accuracy. For these coupling strengths, running the code generates an 'Out of Memory' output in MATLAB. The figures below for $\lambda=1.0 \& 2.0$ were generated with a 50 by 50 knot domain and the optimization was incomplete. Although unoptimized, the bicubic spline surface appears to capture the surface generated by numerical integration of the full system as shown in figures (3) and (4)below.

\section{Results}

We present here the results of running our code for four different values of the coupling parameter. In each case, the error trends were computed between the numerically integrated angle-radial equations, equation (4)using the MATLAB ode45 solver, and the approximate system of phase equations on the torus, given by

$$
\begin{aligned}
\dot{\boldsymbol{\Omega}} & =\mathbf{d}+\lambda \boldsymbol{\Theta}\left(\boldsymbol{\Omega}, \mathbf{r}_{\mathbf{n m}}(\theta, \phi)\right) \\
\mathbf{r} & =\mathbf{r}_{\mathbf{n m}}(\theta, \phi)
\end{aligned}
$$

We set the error tolerance in these computations to $10^{-8}$ and tabulated the results. First, we consider the case $\lambda=0.1$. We ran the code for three different sizes of the knot domain starting with 20 knots in each of the $\theta$ and $\phi$ directions. Figure (1)shows the full and approximate system integration for the case $\lambda=0.1$.
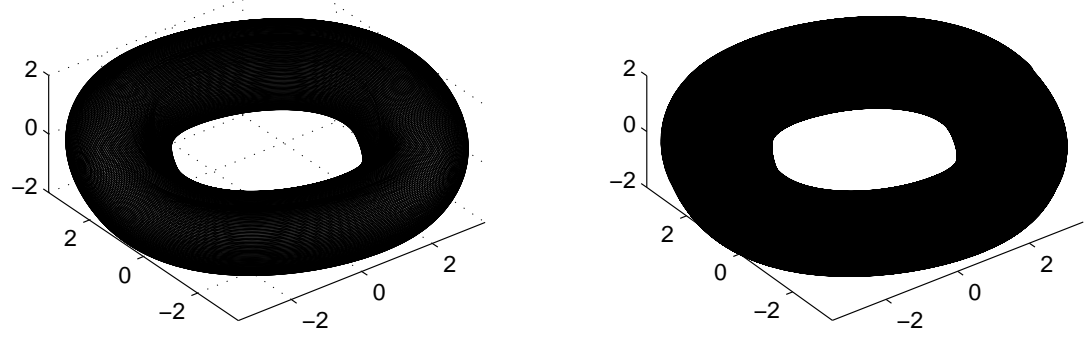

Figure 1: Bicubic approximation (left)and numerically integrated Van der Pol (right) for $\lambda=0.1$ with a 30 by 30 knot domain

Table (3) shows the results of changing the number of knots keeping $\lambda=0.1$ fixed. The third row shows the time $\mathrm{T} 1$ which represents the sum of the seconds it takes to create the necessary matrices 
in the main program plus the time it takes to evaluate once the function called by the optimization subroutine. T2 in seconds is the sum of $\mathrm{T} 1$ and the time it takes to run the optimization subroutine fsolve in MATLAB. The last row gives the normed residual error in each case.

Table 2: Summary table for $\lambda=0.1$ showing timings and residual error.

\begin{tabular}{|c|c|c|c|}
\hline \multicolumn{4}{|c|}{$\lambda=0.1$} \\
\hline Knots & 20 & 30 & 40 \\
\hline T1(seconds) & 0 & 0.0160 & 0.0630 \\
\hline T2(seconds) & 7.0620 & 13.3290 & 38.8750 \\
\hline Normed Residual Error & $1.92718 \mathrm{e}-010$ & $9.35666 \mathrm{e}-010$ & $6.38028 \mathrm{e}-009$ \\
\hline
\end{tabular}

Figure (2) represents the $\lambda=0.6$ case. We observe some deforming of the torus but the bicubic surface appears to be similar to that generated by the MATLAB's ode45 solver.
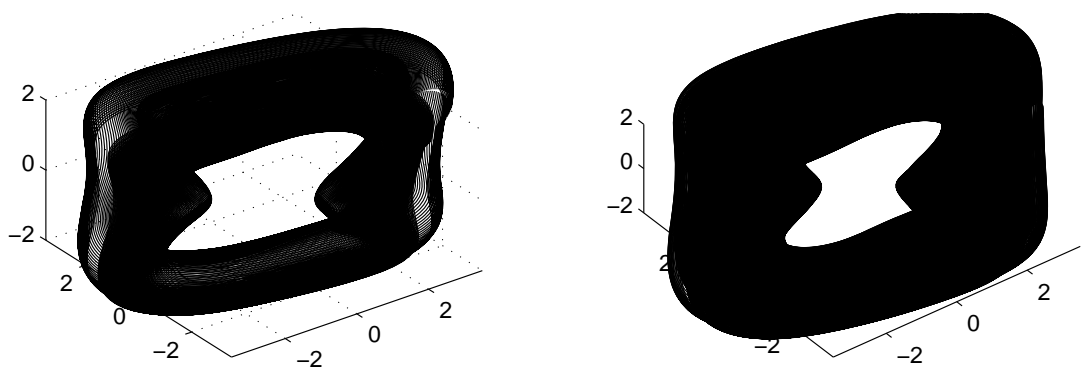

Figure 2: Bicubic approximation (left)and numerically integrated Van der Pol (right) for $\lambda=0.6$ with a 45 by 45 knot domain

Table 3: Summary table for $\lambda=0.6$ showing timings and residual error.

\begin{tabular}{|c|c|c|c|}
\hline \multicolumn{4}{|c|}{$\lambda=0.6$} \\
\hline Knots & 40 & 45 & 50 \\
\hline T1(seconds) & 0.0620 & 0.0780 & 0.1410 \\
\hline T2(seconds) & 68.1250 & 80.7190 & 265.6710 \\
\hline Normed Residual Error & $8.00207 \mathrm{e}-011$ & $9.18135 \mathrm{e}-010$ & $7.72686 \mathrm{e}-008$ \\
\hline
\end{tabular}

As mentioned in the previous section, for values of $\lambda \geq 1.0$ the optimization did not terminate successfully due to insufficient number of knots. Figures (3) and (4) depict two such cases. We note that as the strength of coupling increase, the inner sides of the invariant torus get closer and eventually connect with each other. Again, the results appear to be consistent.

\section{Conclusions}

This case study presents an alternate approach to approximating the invariant torus of the coupled Van der Pol oscillator. The bicubic B-spline collocation method in conjunction with the vectorization capabilities offered by the MATLAB processing system provides us with the tools for developing a significant time saving optimization algorithm for efficiently computing the approximate periodic 

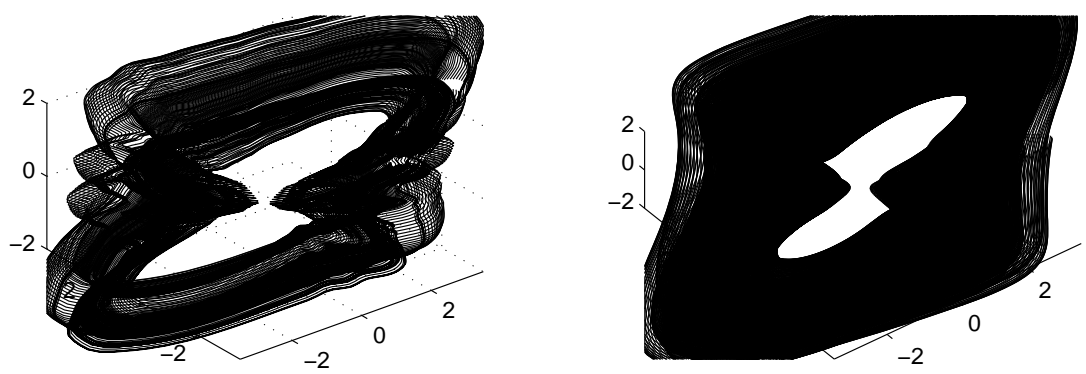

Figure 3: Bicubic approximation (left)and numerically integrated Van der Pol (right) for $\lambda=1.0$ with a 50 by 50 knot domain

surface. Comparisons of the present approach with the MATLAB numerical integration of the full system of coupled oscillators indicate that the method demonstrated in this paper is promising. For strong nolinear coupling, computer memory appears to be the only limitation.

\section{Disclaimer}

Certain commercial software products are identified in this paper in order to adequately specify the computational procedures. Such identification does not imply recommendation or endorsement by the National Institute of Standards and Technology nor does it imply that the software products identified are necessarily the best available for the purpose.

\section{References}

[1] Bogoliubov, N. N., Mitropolsky, Y. A., Asymptotic Methods in the Theory of Non-Linear Oscillations, Gordon and Breach, New York, 1961.

[2] Dag, I., Irk, D.,and Sahin, A., 'B-spline Collocation Methods for Numerical Solutions of the Burger's Equation', Mathematical Problems in Engineering 5, 2005, 521-538.

[3] Dyksen, W., Houstis, E., Lynch, R., and Rice, J.,'The performance of the collocation and Galerkin methods with Hermite bicubics',SIAM Journal of Numerical Analysis 21, 1984, 695715 .

[4] Ge, T., and Leung, A. Y. T.,'Construction of Invariant Torus Using Toeplitz Jacobian Matrices/Fast Fourier Transform Approch', Nonlinear Dynamics 15, 1998, 283-305.

[5] Gilsinn, D. E., 'Asymptotic Approximations of Integral Manifolds', SIAM J. Appl. Math., 47, 5, 1987, 929-940.

[6] Gilsinn,D.E., 'Constructing Invariant Tori for Two Weakly Coupled van der Pol Oscillators', Nonlinear Dynamics 4, 1993, 289-308. 

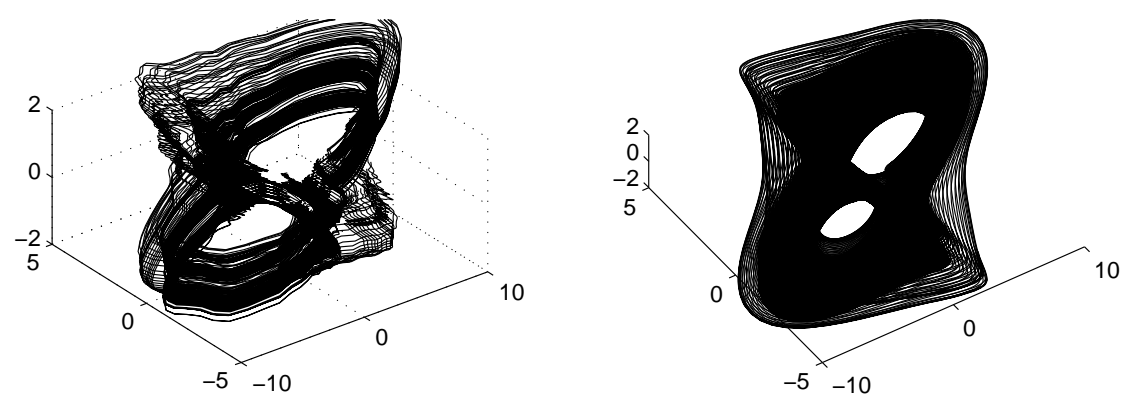

Figure 4: Bicubic approximation (left)and numerically integrated Van der Pol (right) for $\lambda=2.0$ with a 50 by 50 knot domain

[7] Gilsinn,D.E., 'Constructing Galerkin's Approximations of Invariant Tori Using MACSYMA', Nonlinear Dynamics 8, 1995, 269-305.

[8] Guckenheimer, J., and Holmes, P., Nonlinear Oscillations, Dynamical Systems, and Bifurcations of Vector Fields, Springer-Verlag, New York, 1983, 130 -138.

[9] Hale, J. K., 'Integral manifolds of perturbed differential equations', Ann. of Math., 73, 1961, 496-531.

[10] Hale, J. K., Ordinary Differential Equations, Wiley-Interscience, New York, 1969.

[11] Lu,F., and Milios, E. E., 'Optimal spline fitting to planar shape',Signal Processing 37, 1994,129140 .

[12] Prenter,P.M., Splines and Variational Methods, John Wiley \& Sons, 1975, 78 - 87.

[13] Wiggins,S., Introduction to Applied Nonlinear Dynamical Systems and Chaos, Springer-Verlag, New York, 1990, 193 - 204.

\section{A B-spline Code for Approximate Torus}

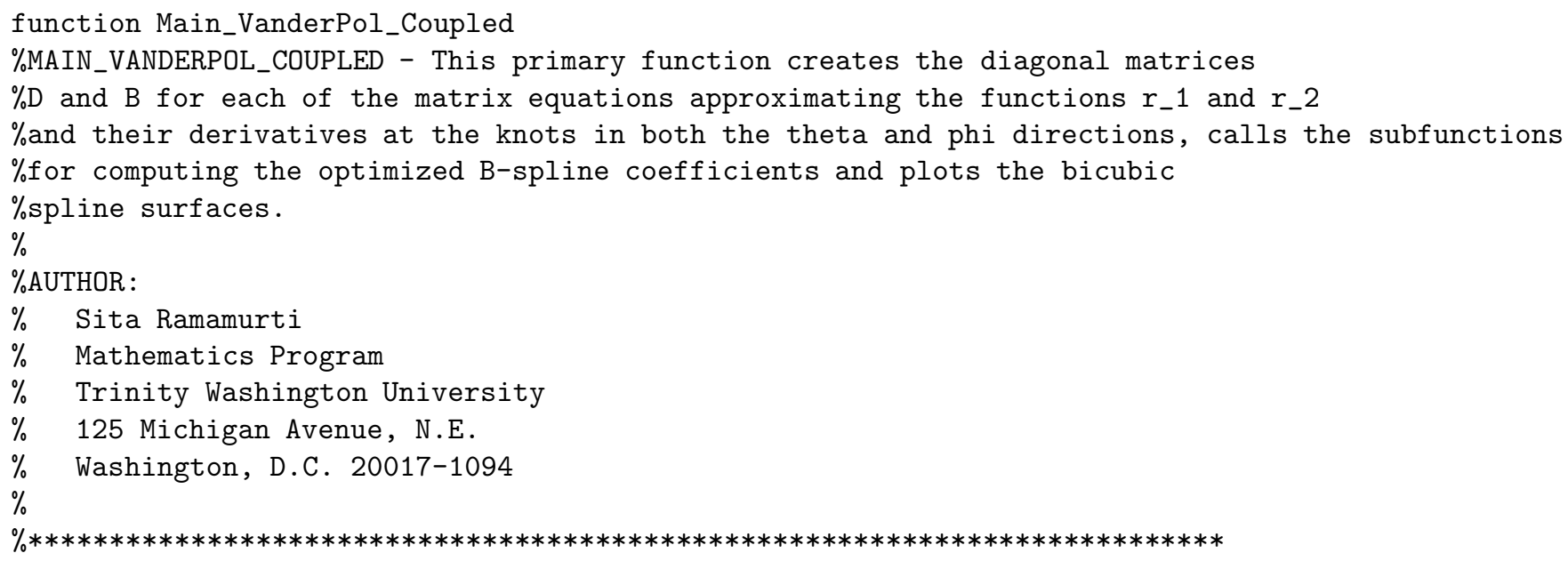


global lambda meu1 meu2 a alph

global $\mathrm{m} n \mathrm{~h}$ k delta1 delta2 $\mathrm{nm} \mathrm{np} 1 \mathrm{mp} 1 \mathrm{np} 1 \mathrm{mp} 1 \mathrm{mp} 4 \mathrm{np} 3 \mathrm{np} 2 \mathrm{np} 4 \mathrm{mp} 3 \mathrm{mp} 2 \mathrm{np} 3 \mathrm{mp} 3 \mathrm{np} 5 \mathrm{mp} 5$

global theta phi thetavector phivector

global r1_nm dr1dtheta_nm dr1dphi_nm

global r2_nm dr2dtheta_nm dr2dphi_nm

global thetadot phidot

global r1dot r2dot

global R1 R2

global B1red B2red B3red Jpat Jred

global c d

global tim2 tim3

$\%$ enter the following parameters

meu1=1.0; $\%$ input ('enter the parameter meu1, meu1=');

meu2=1.414; $\%$ input ('enter the parameter meu2, meu2=');

$\mathrm{a}=0.2 ; \%$ input ('enter the parameter $\mathrm{a}, \mathrm{a}=$ ') ;

alph=0.4;\%input ('enter the parameter alph,alph=');

\%enter the following inputs

lambda=input ('enter the parameter lamda,lambda=');

$\mathrm{n}=$ input ('enter harmonics $\mathrm{n}, \mathrm{n}=$ ') ;

$\mathrm{m}=$ input ('enter harmonics $\mathrm{m}, \mathrm{m}=$ ') ;

$\mathrm{np} 1=\mathrm{n}+1 ; \mathrm{mp} 1=\mathrm{m}+1 ; \mathrm{np} 2=\mathrm{n}+2 ; \mathrm{mp} 2=\mathrm{m}+2 ; \mathrm{np} 3=\mathrm{n}+3 ; \mathrm{mp} 3=\mathrm{m}+3$;

$\mathrm{np} 1 \mathrm{mp} 1=\mathrm{np} 1 * \mathrm{mp} 1 ; \mathrm{np} 3 \mathrm{mp} 3=\mathrm{np} 3 * \mathrm{mp} 3$;

$\mathrm{nm}=\mathrm{n} * \mathrm{~m}$;

tim=clock;

theta $=\operatorname{zeros}(\mathrm{np} 3,1)$;

phi=zeros $(\mathrm{mp} 3,1)$;

B1red=zeros $(\mathrm{nm}, \mathrm{nm})$;

B2red $=$ zeros $(\mathrm{nm}, \mathrm{nm})$;

B3red $=\operatorname{zeros}(\mathrm{nm}, \mathrm{nm})$;

Jpat $=\operatorname{zeros}(2 * \mathrm{~nm}, 2 * \mathrm{~nm})$;

$\%$ mesh specified by $\{$ theta $=$ meu1*omega1_i:i=1,2, $\ldots, n+5\} \&\{$ phi=meu2*omega2_j:i=1,2, $\ldots, m+5\}$

delta1=2*pi/n;

$\operatorname{delta} 2=2 * \mathrm{pi} / \mathrm{m}$;

$\mathrm{h}=2: \mathrm{np} 2$;

theta $(\mathrm{h})=(\mathrm{h}-2) * \operatorname{delta} 1$;

$\mathrm{k}=2: \mathrm{mp} 2$;

phi $(\mathrm{k})=(\mathrm{k}-2) * \operatorname{delta} 2$;

$\%$ creating the B1redmatrix

$\%$ create the matrix block B1 


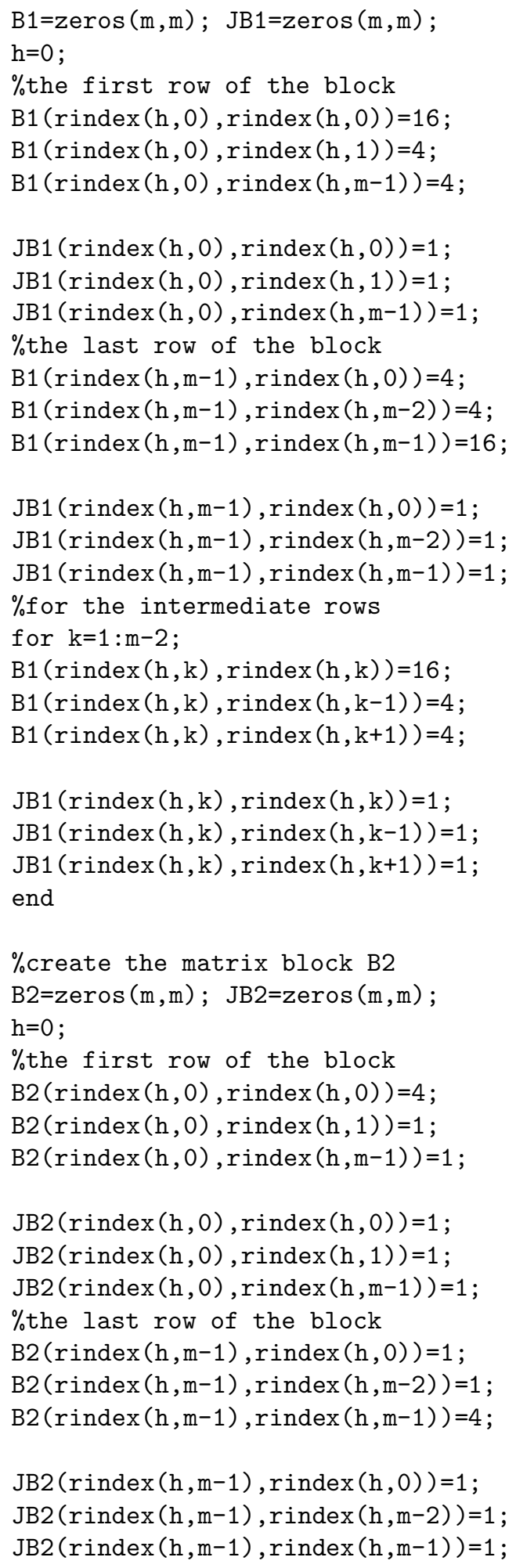




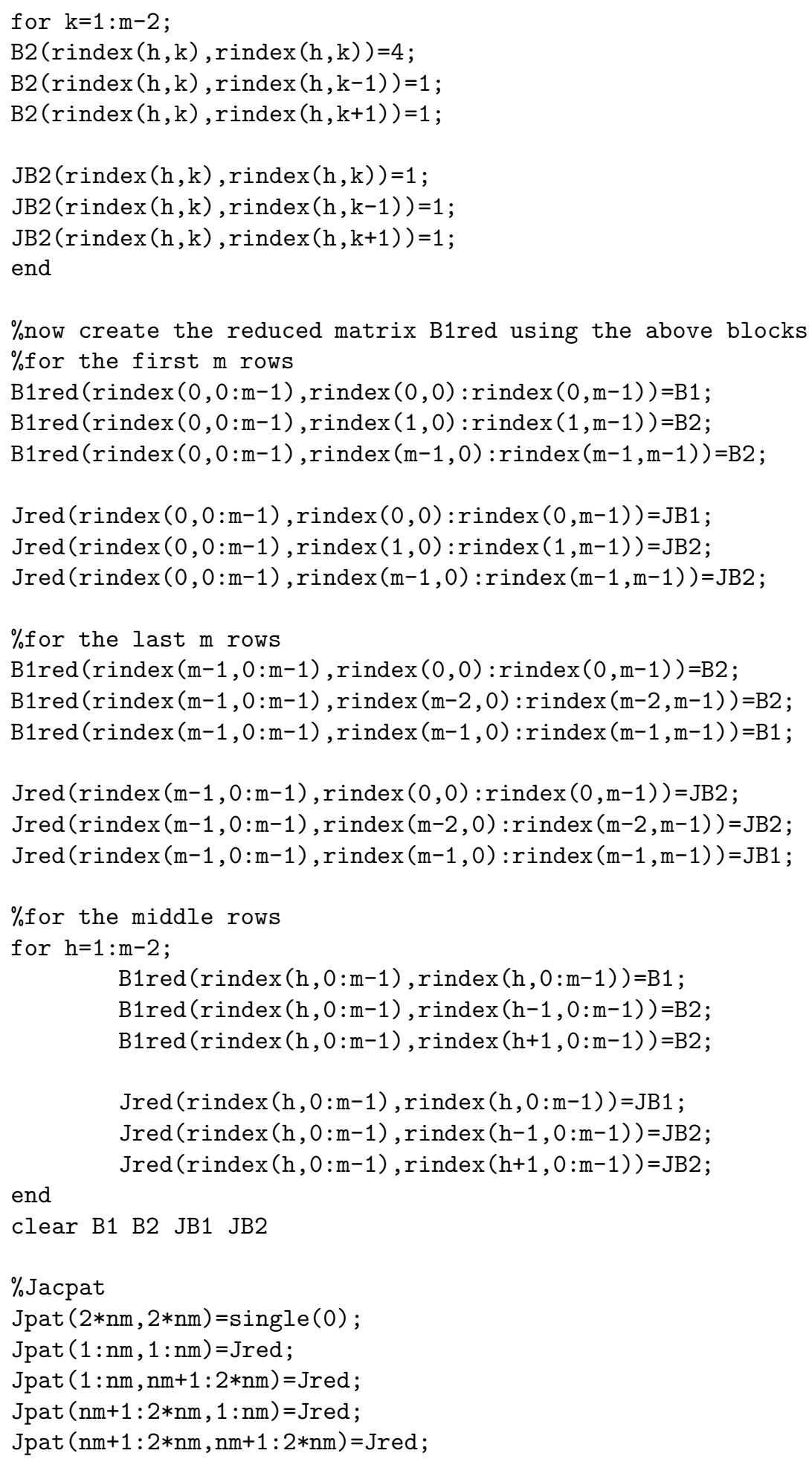




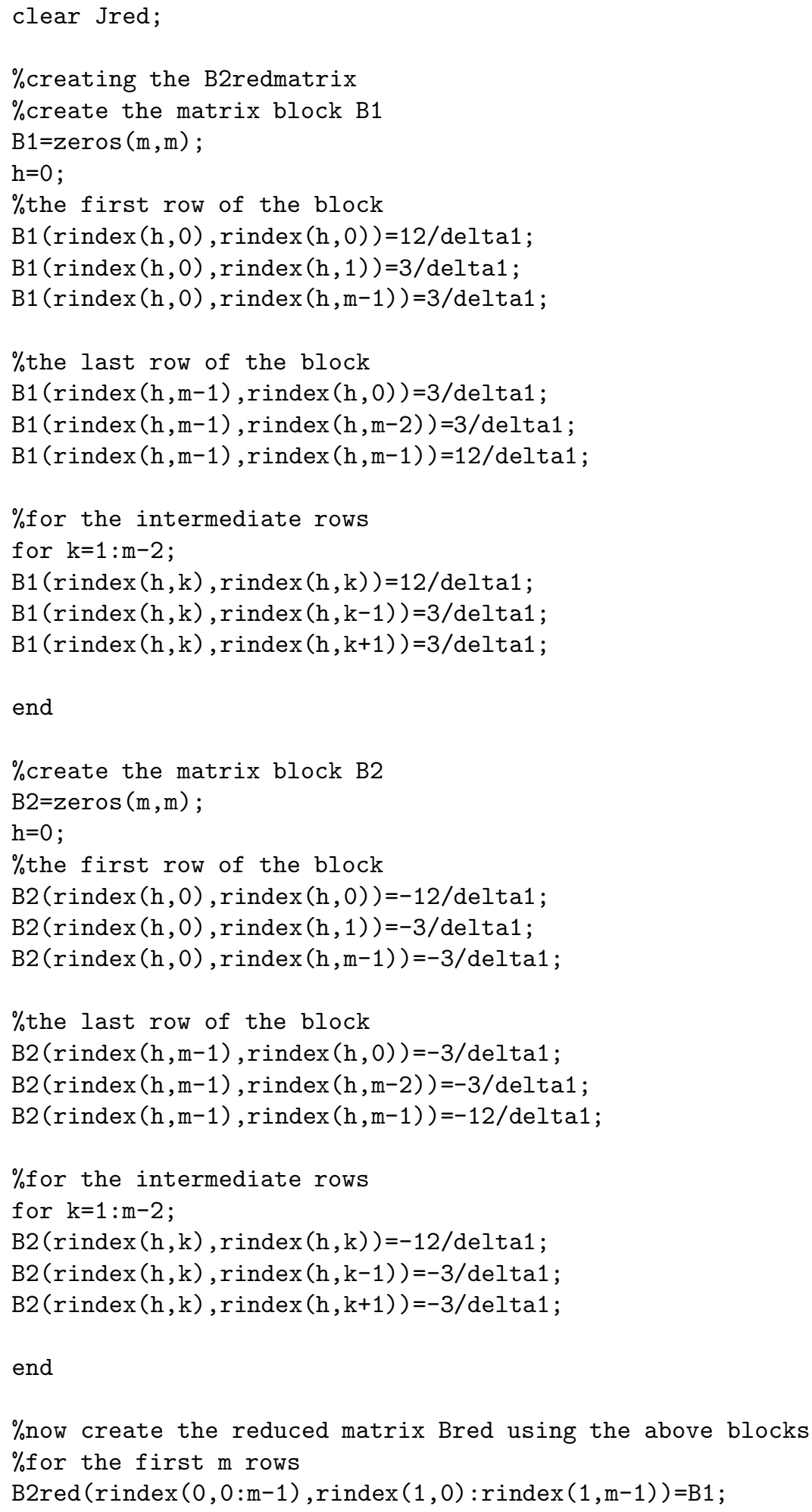




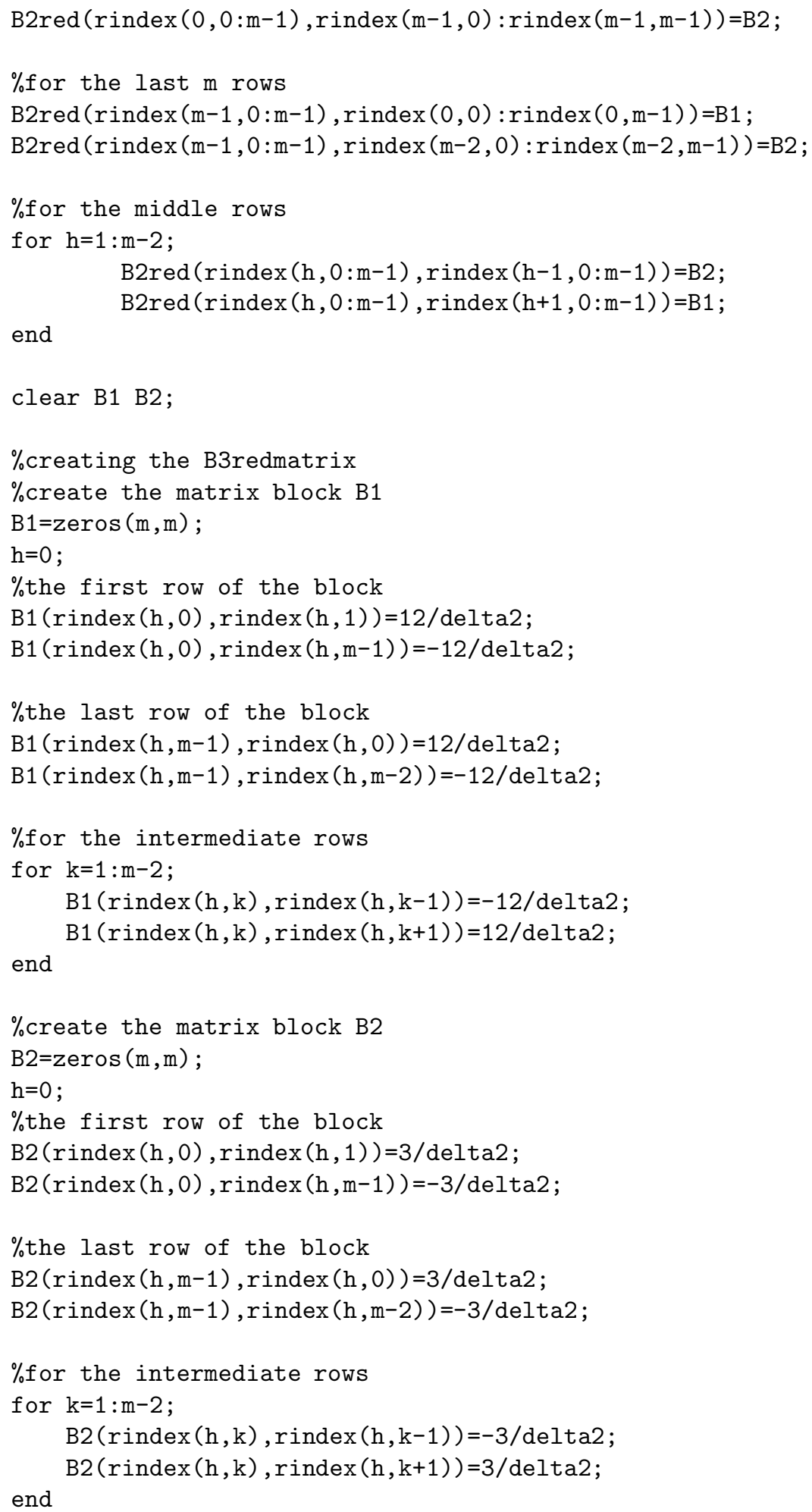




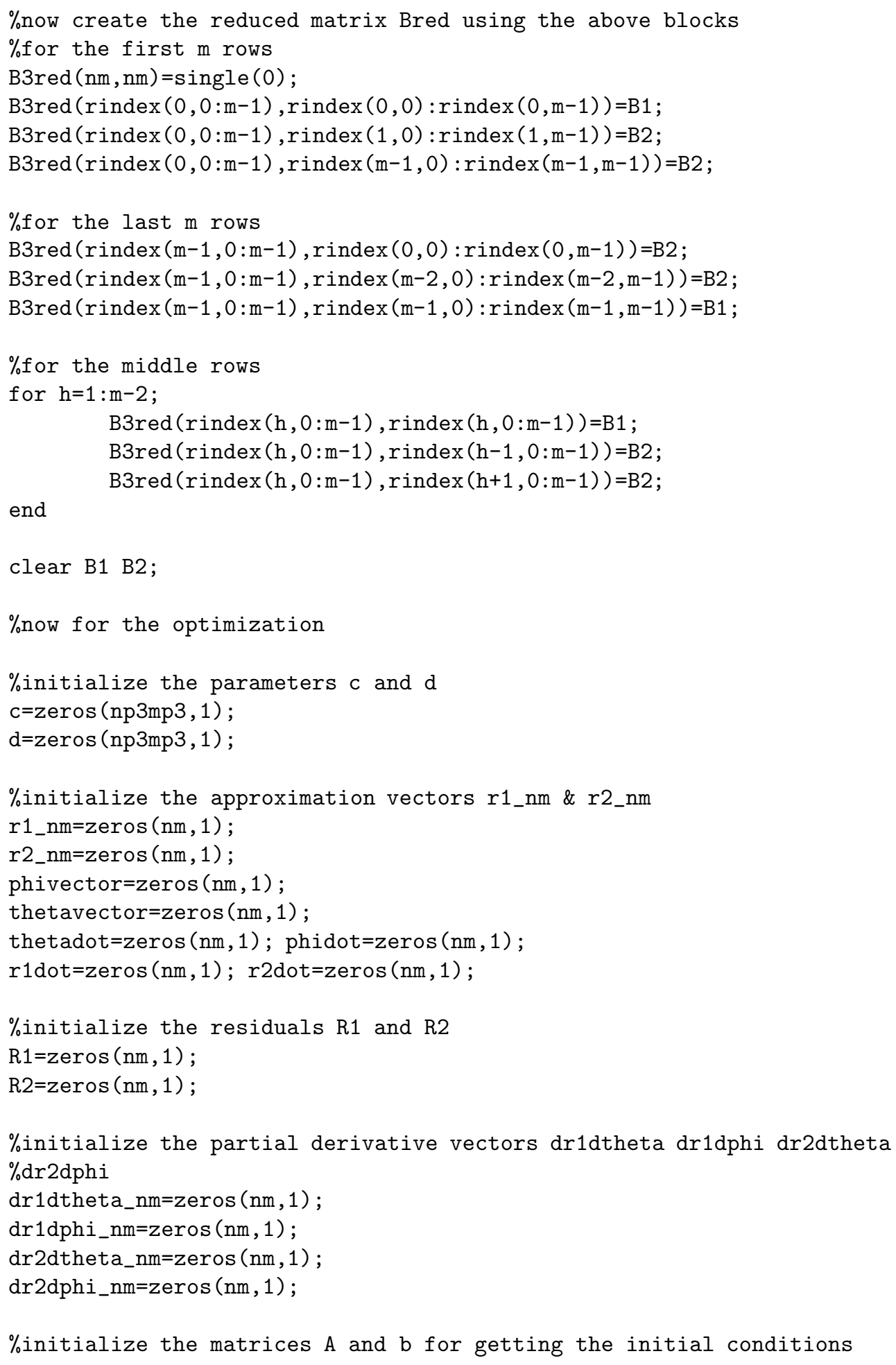




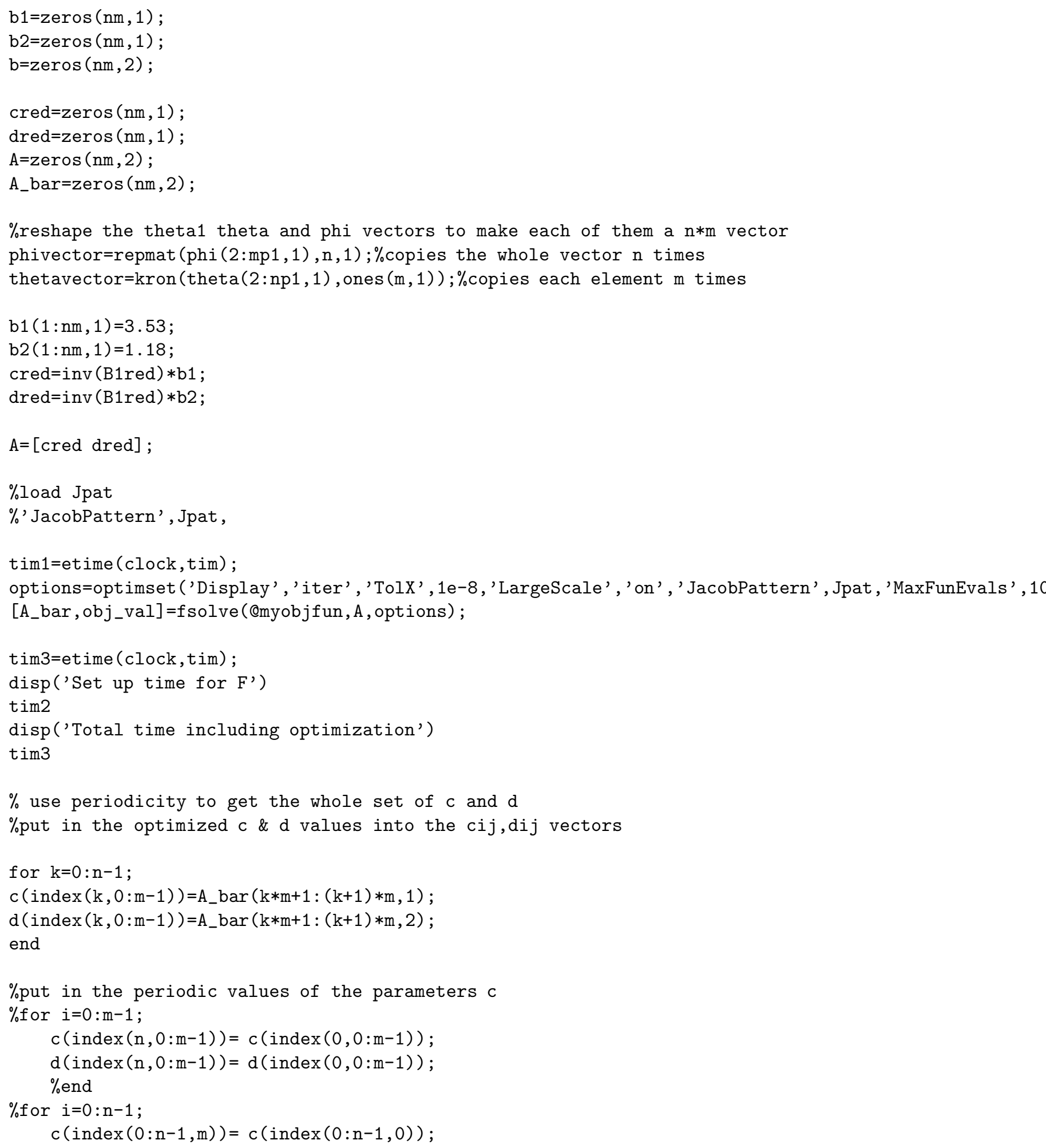




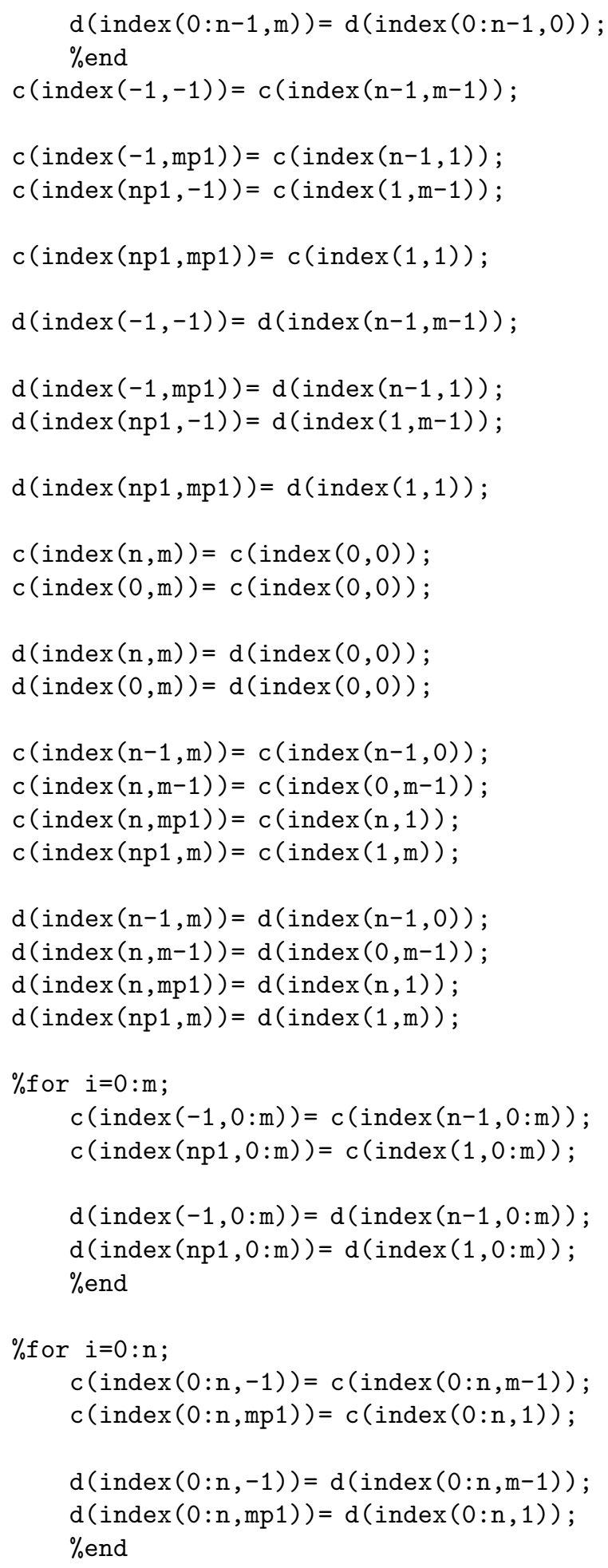




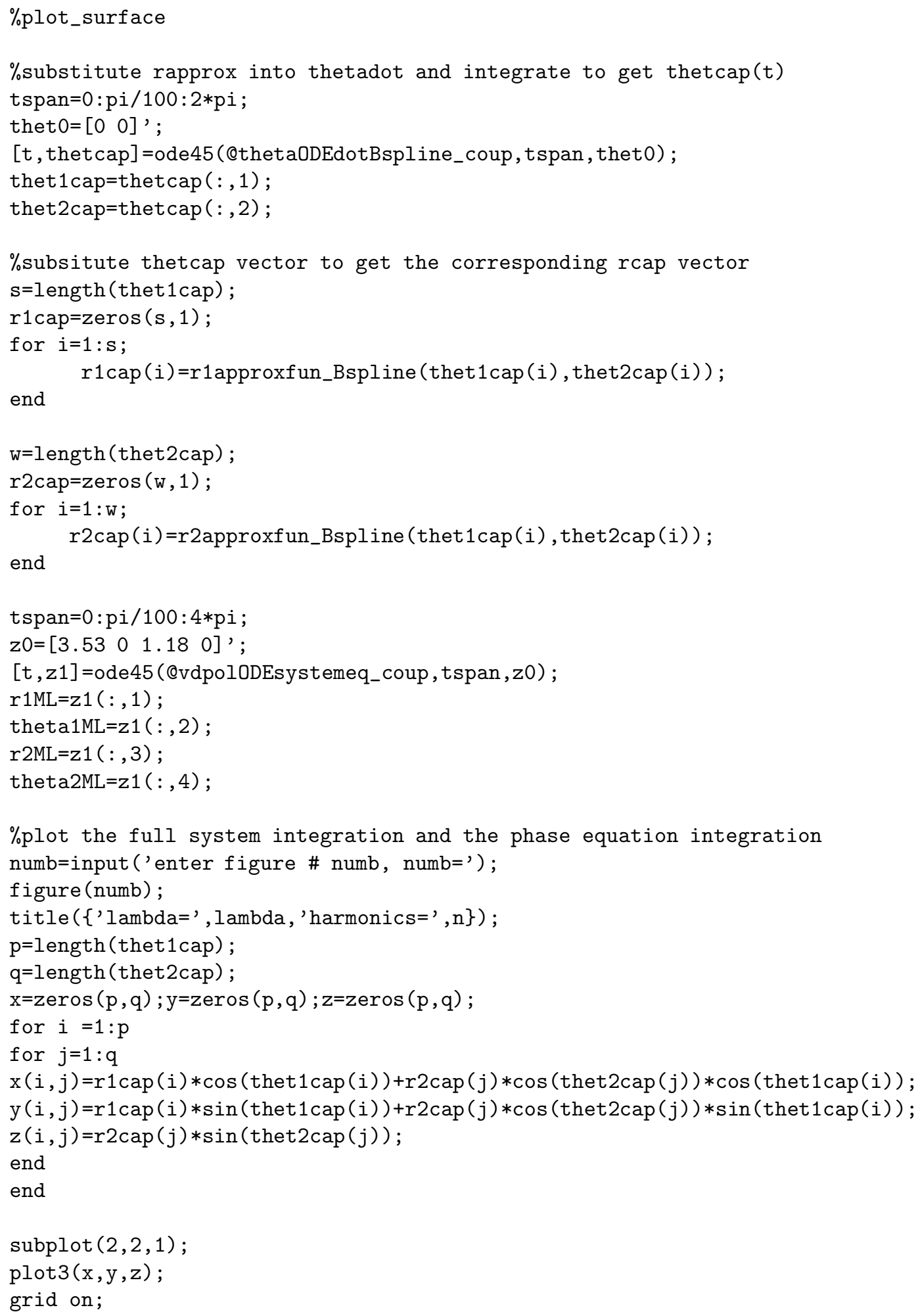




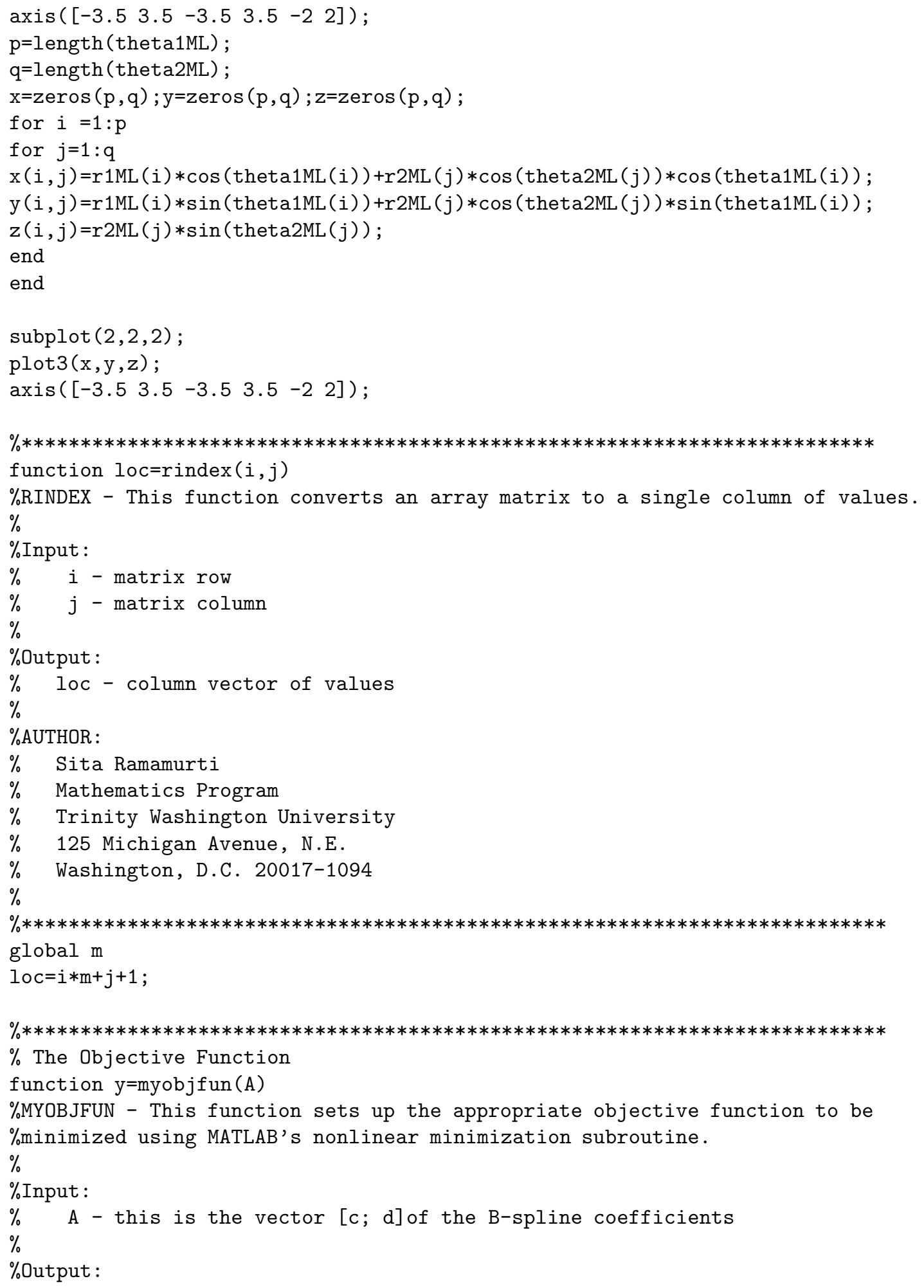




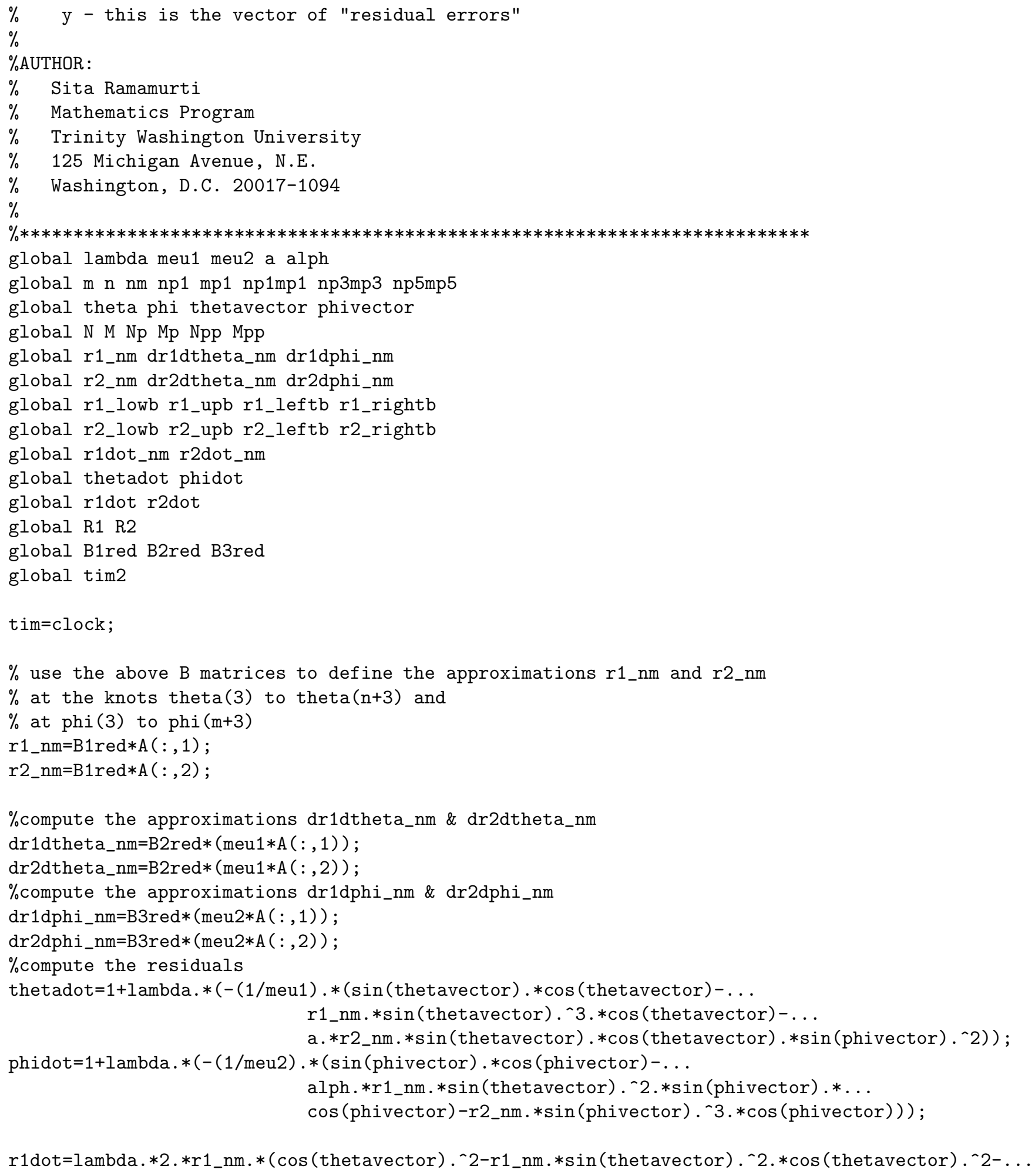




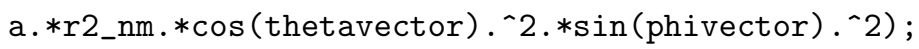

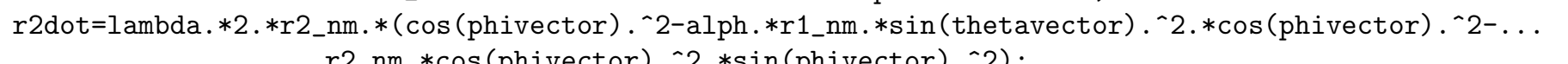

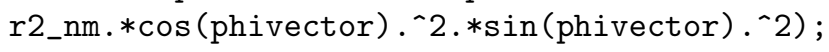

$\%$ compute the r1\&r2dot matrices and the residuals

r1dot_nm=dr1dtheta_nm.*thetadot+dr1dphi_nm.*phidot;

r2dot_nm=dr2dtheta_nm.*thetadot+dr2dphi_nm. *phidot;

$\mathrm{R} 1=r 1 d o t \_n m-r 1 d o t ;$

R2=r2dot_nm-r2dot;

$y=[R 1 ; R 2] ;$

tim2=etime (clock, tim);

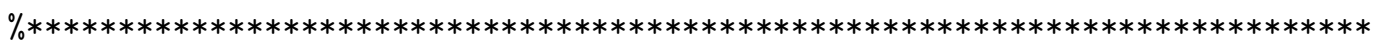

function loc $=$ index $(i, j)$

$\%$ INDEX - This function converts an array matrix to a single column of values.

$\%$

$\%$ Input :

$\% \quad i-m a t r i x$ row

$\% \quad j-$ matrix column

$\%$

$\%$ Output:

$\%$ loc - column vector of values

$\%$

$\%$ AUTHOR :

$\% \quad$ Sita Ramamurti

$\% \quad$ Mathematics Program

$\% \quad$ Trinity Washington University

$\% 125$ Michigan Avenue, N.E.

$\%$ Washington, D.C. 20017-1094

$\%$

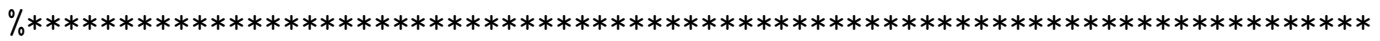

global mp3

$10 c=(i+1) * m p 3+2+j$;

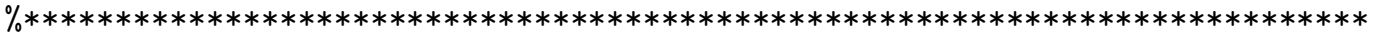

$\%$ integration of the phase equation to get thetacap

function thetaprime=thetaODEdotBspline_coup ( $t$, thet)

\%THETAODEDOTBSPLINE - This function substitutes the bicubic spline

\%approximations for $r_{-} 1$ and $r_{-} 2$ into the Van der Pol system equations for the derivatives $\%$ of omega_1 and omega_2.

$\%$ Input:

$\% \quad t-a$ vector specifying the interval of integration

$\%$ thet - a vector specifying the knot location in the theta and phi

$\%$ directions

$\%$

$\%$ Output:

$\%$ thetaprime - vector of derivatives of omega_1 and omega_2 


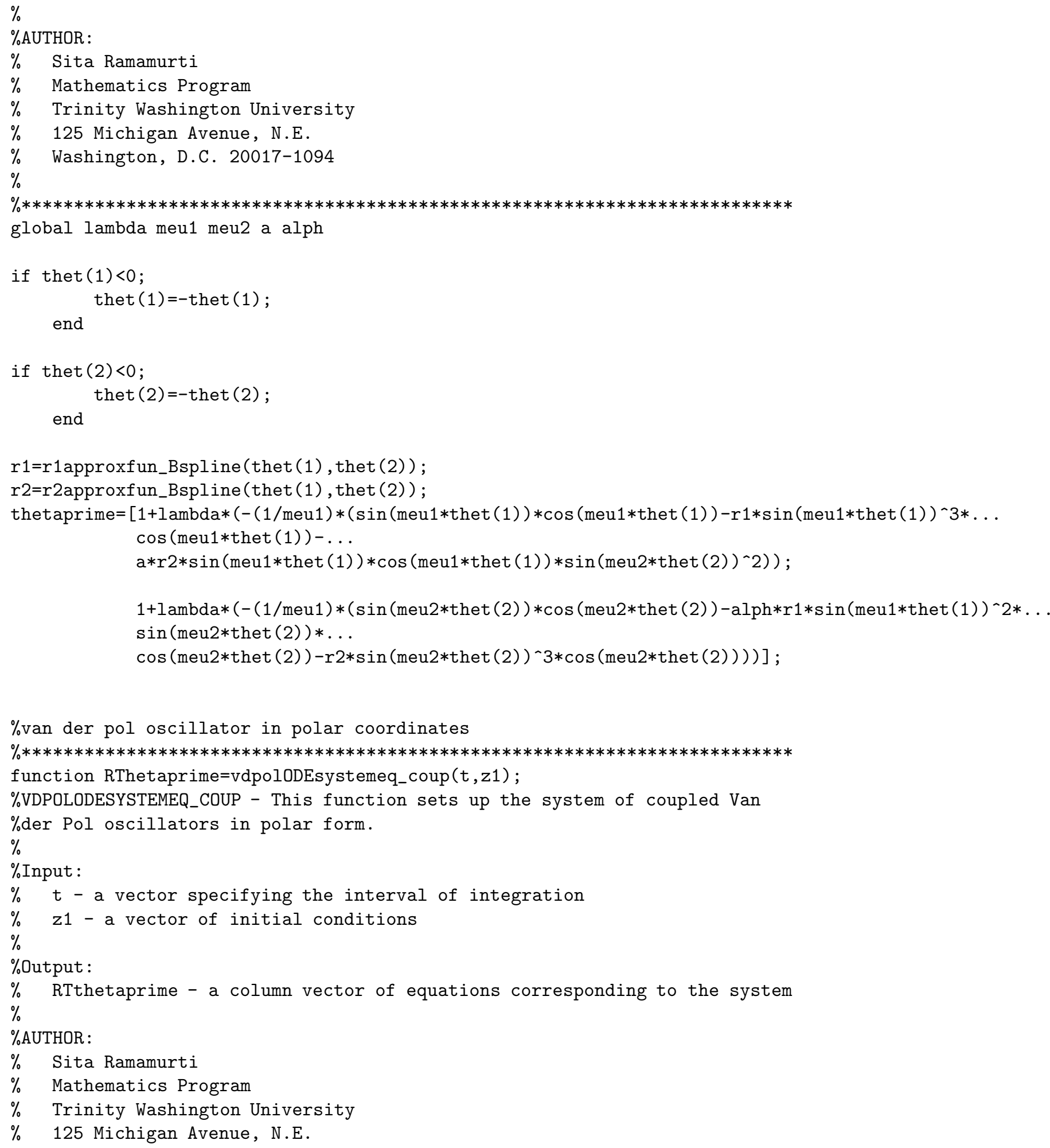




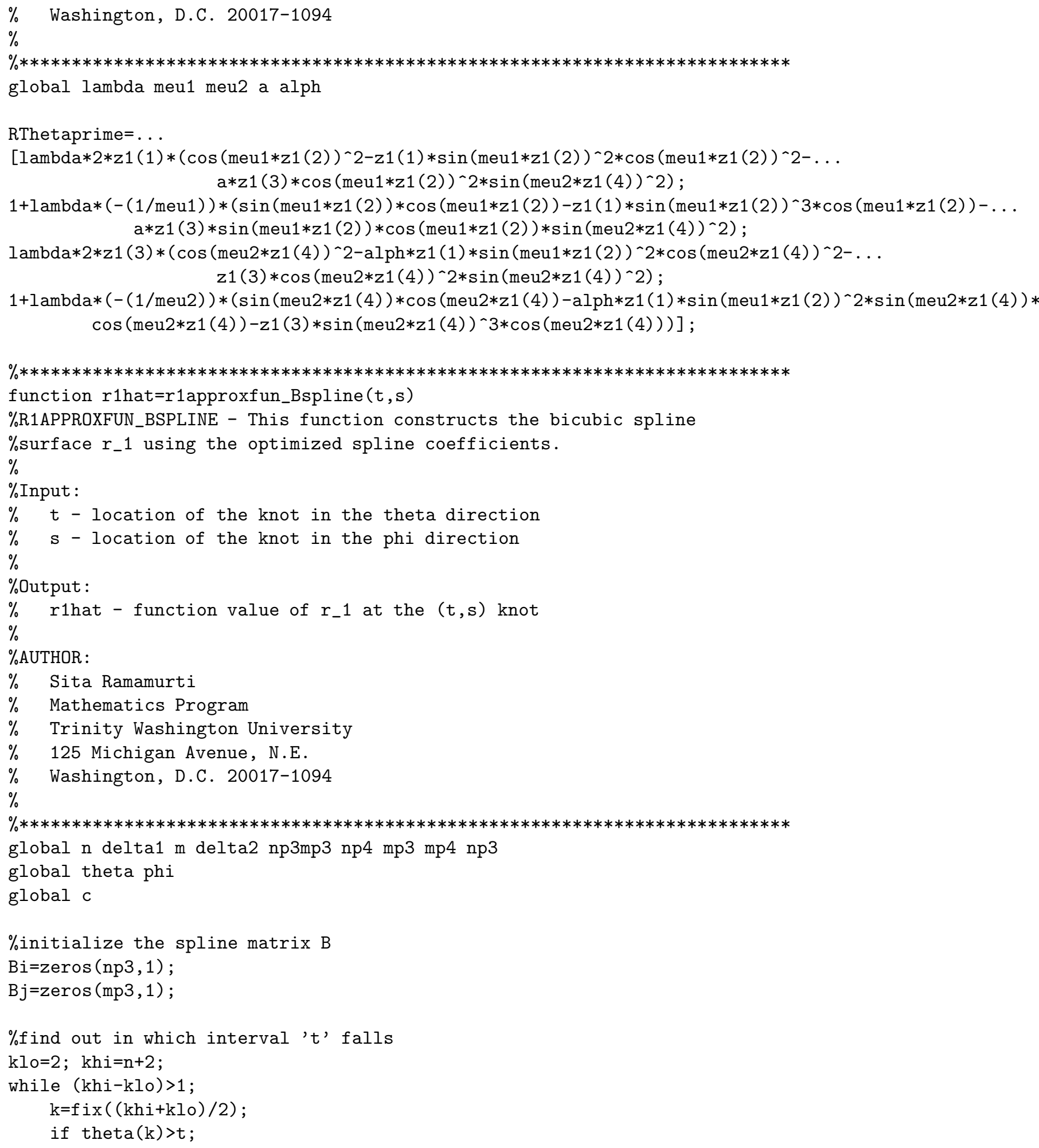




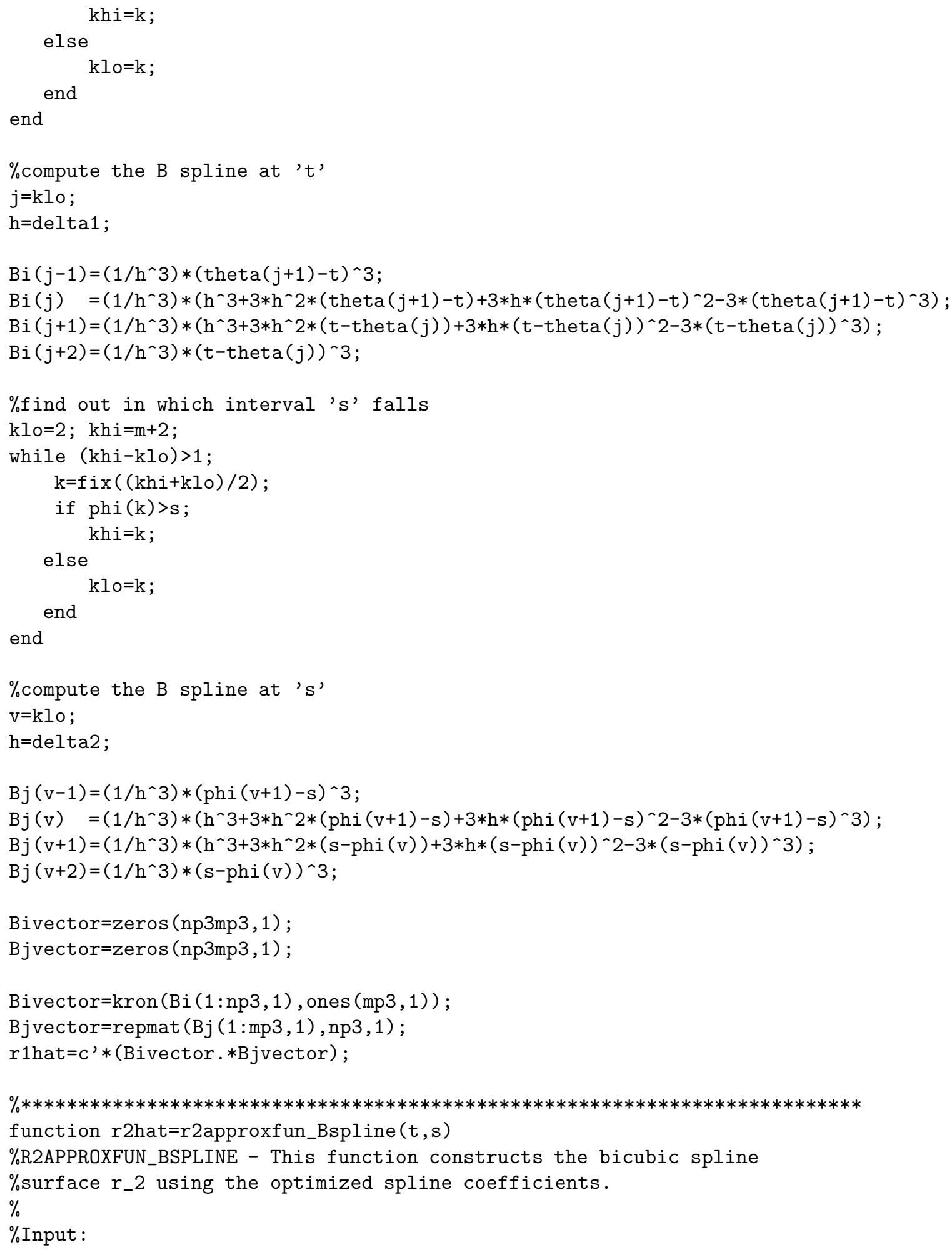




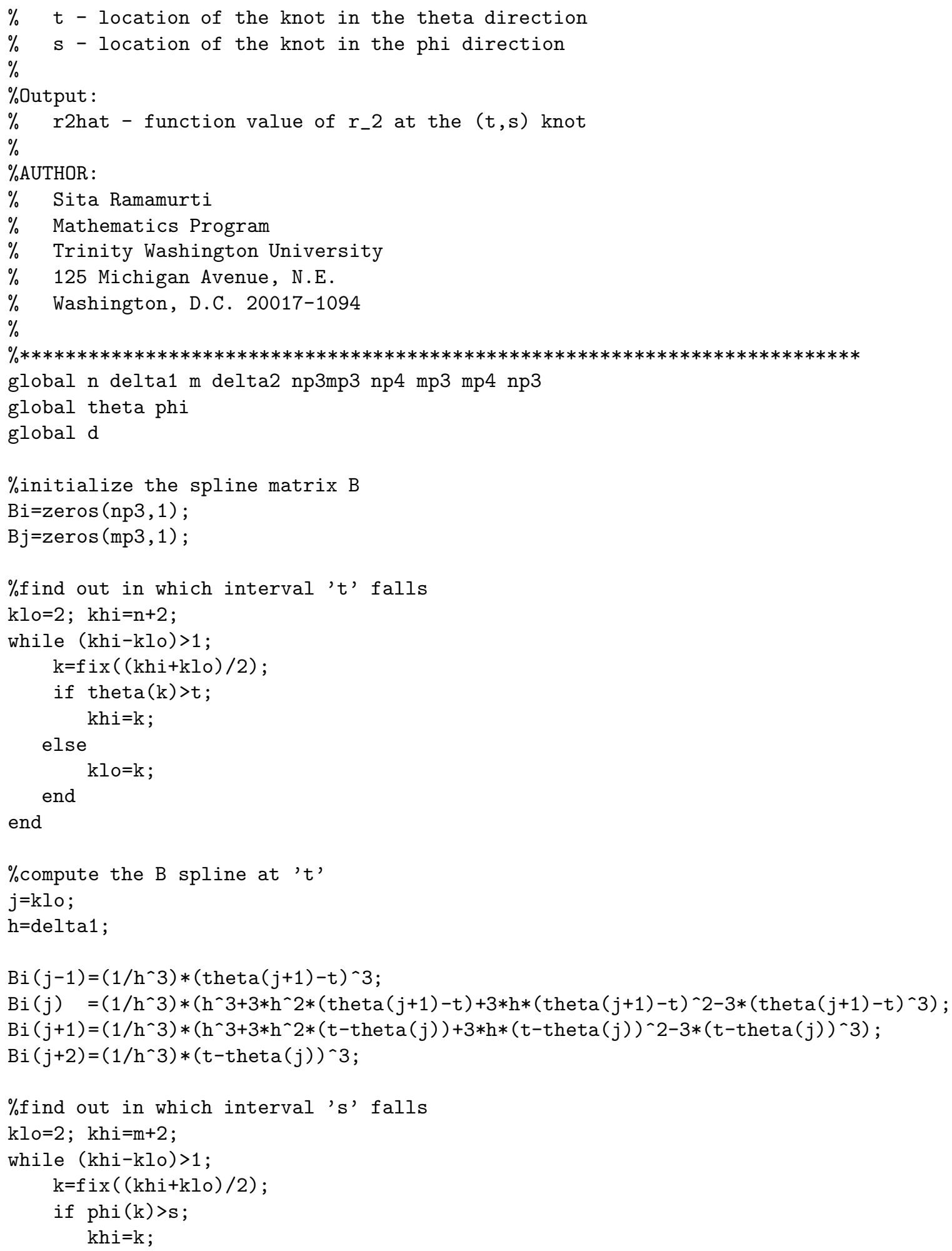




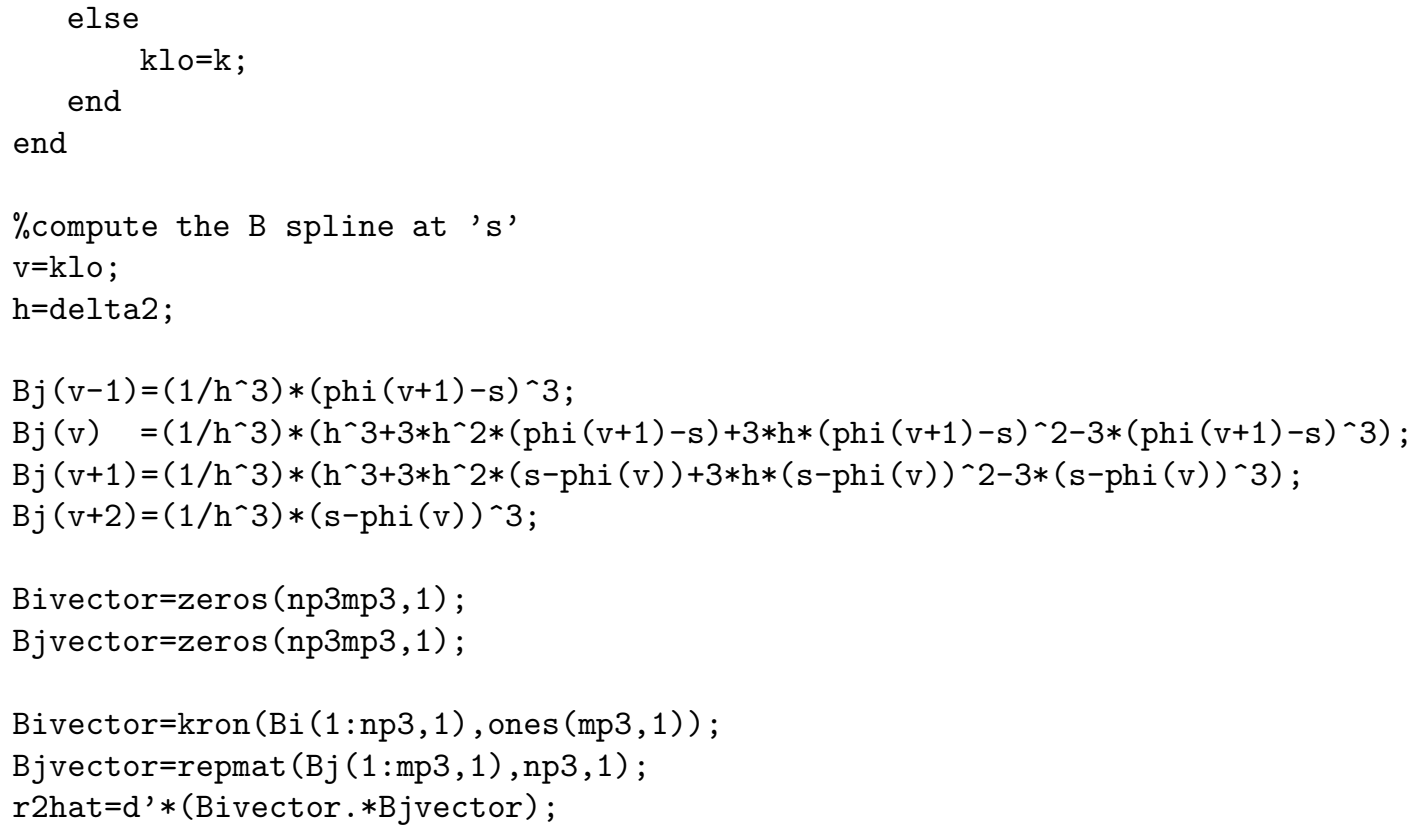

